\title{
Grenzwerte für absolute Fahruntüchtigkeit bei Radfahrern
}

Thomas Daldrup

Benno Hartung

Kurt Rüdiger Maatz

Nona Mindiashvili

Eckhard H. Roth

Holger Schwender

Unfallforschung der Versicherer

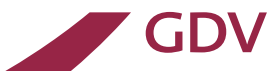




\title{
Grenzwerte für absolute Fahruntüchtigkeit bei Radfahrern
}

\author{
Prof. Dr. Thomas Daldrup \\ Dr. Benno Hartung \\ Richter BGH i. R. Kurt Rüdiger Maatz \\ Nona Mindiashvili M. A. \\ Dr. Dr. Eckhard H. Roth \\ Prof. Dr. Holger Schwender
}

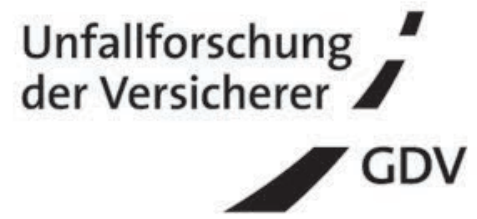




\section{Impressum}

\section{Gesamtverband der Deutschen Versicherungswirtschaft e. V.}

\section{Unfallforschung der Versicherer}

Wilhelmstraße 43/43G, 10117 Berlin

Postfach 0802 64, 10002 Berlin

E-Mail: unfallforschung@gdv.de

Internet: www.udv.de

Facebook: www.facebook.com/unfallforschung

Twitter: @unfallforschung

YouTube: www.youtube.com/unfallforschung

ISBN-Nr.: 978-3-939163-56-5

Redaktion: Dr.-Ing. Axel Malczyk

Bildnachweis: UDV und siehe Quellenangaben

Erschienen: 08/2014 


\section{Grenzwerte für absolute Fahruntüchtigkeit bei Radfahrern}

bearbeitet durch:

Institut für Rechtsmedizin, Universitätsklinikum Düsseldorf

Prof. Dr. Thomas Daldrup

Dr. Benno Hartung

Richter BGH i. R. Kurt Rüdiger Maatz

Nona Mindiashvili M.A.

Dr. Dr. Eckhard H. Roth

Prof. Dr. Holger Schwender

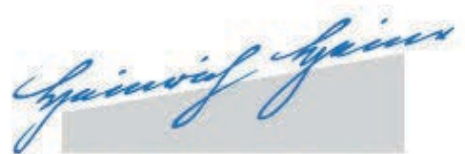

HEINRICH HEINE

UNIVERSITÄT DÜSSELDORF

Bei der UDV betreut von:

Dr.-Ing. Axel Malczyk

\section{Unfallforschung} der Versicherer 

INHALTSVERZEICHNIS

1. Ziel des Projekts 4

2. Versuchsaufbau 5

2.1 Allgemeiner Versuchsaufbau 5

2.2 Maßnahmen zur Verhinderung von Unfällen und Verletzungen 10

$\begin{array}{ll}\text { 2.3 Praktische Anordnung der Versuche } & 10\end{array}$

2.4 Versicherung 14

2.5 Drogenscreening 14

$\begin{array}{ll}\text { 2.6 Datenschutz } & 14\end{array}$

3. Material und Methodik 15

3.1 Fahrräder 15

$\begin{array}{ll}3.2 \text { Schutzausrüstung } & 16\end{array}$

3.3 Atemalkoholtestgerät Dräger Alcotest 9510 DE 16

3.4 Ärztliche Untersuchung im Rahmen der Blutentnahmen 17

$\begin{array}{ll}\text { 3.5 Augenärztliche Untersuchungen } & 17\end{array}$

3.6 Alkoholika 19

$\begin{array}{ll}3.7 \text { Videokameras } & 20\end{array}$

3.8 Übernachtung $\quad 20$

$\begin{array}{ll}3.9 \text { Auswertung } & 20\end{array}$

$\begin{array}{ll}\text { 4. Probanden } & 21\end{array}$ 
5. Ergebnisse 22

5.1 Ergebnisse des Drogen- und Arzneimittelscreenings 22

5.2 Erreichte Blutalkoholkonzentrationen (BAK) 23

5.3 Ergebnisse der ärztlichen Untersuchungen im Rahmen der Blutentnahmen $\quad 26$

5.4 Ergebnisse der augenärztlichen Untersuchungen 33

5.5 Ergebnisse der Videoanalysen der Fahrversuche 35

5.6 Überprüfung der Robustheit 44

5.7 Gesamtleistungsvermögen $\quad 46$

$\begin{array}{ll}\text { 6. Diskussion } & 47\end{array}$

$\begin{array}{ll}\text { 7. Zusammenfassung } & 50\end{array}$

8. Summary 51

9. Anlage: Juristische Würdigung der Versuchsergebnisse 52

10. Literatur $\quad 54$

11. Danksagung 55 
Die bisherigen Erkenntnisse und die gültige Rechtsprechung zur alkoholbedingten Fahruntüchtigkeit von Fahrradfahrern basieren im Wesentlichen auf den von Schewe et al. durchgeführten Experimenten [1, 2]. Hierbei wurden vergleichende Untersuchungen zwischen Nüchternleistungen und Leistungen bei Blutalkoholkonzentrationen von etwa $0,8 \%$ o und etwa $1,3 \%$ an 71 Probanden im Alter zwischen 18 und 38 Jahren durchgeführt. Folgende Fahraufgaben waren von den Probanden zu bewältigen: Kreisfahrten bei Kreisdurchmessern von 6 bzw. 8 m, Geradeausfahrt auf einer sich verjüngenden Fahrspur von 45 m Länge, Durchfahren von acht seitenversetzten Toren, Slalomfahrt durch in Linie aufgestellte Kegel mit zunehmend geringerem Abstand. Die Versuche erfolgten dabei jeweils ab 9 Uhr morgens, also ausschließlich bei Tageslicht. Eine weitere methodische Einschränkung stellte die Tatsache dar, dass keine Brems- und Anhalteversuche und kein Screening auf Arzneimittel und Drogen durchgeführt wurden. Eine grundsätzliche, methodisch kaum vermeidbare Schwäche ergab sich daraus, dass die Probanden im Rahmen der gewählten Versuchsanordnung in der Regel besonders motiviert und konzentriert waren und die Versuchsanordnung den Probanden bereits vorher bekannt war.

Die im Rahmen der vorliegenden Studie geplanten Untersuchungen sollten die Frage beantworten, ab welchem Alkoholisierungsgrad von absoluter Fahruntüchtigkeit auf dem Fahrrad auszugehen ist. Insbesondere war zu klären, ob der derzeit gemäß aktueller Rechtsprechung festgelegte Grenzwert der Blutalkoholkonzentration (BAK) von 1,6\%o zur Annahme einer absoluten Fahruntüchtigkeit geeignet ist. Dazu sollten erneut Probandenversuche durchgeführt werden, wobei der Alkoholkonsum und die Fahrtests unter realistischeren Bedingungen als bei Schewe stattfinden und die medizinischen Untersuchungen deutlich ausgeweitet werden sollten. 


\section{VERSUCHSAUFBAU}

\subsection{Allgemeiner Versuchsaufbau}

\subsubsection{Grundsätzliche Überlegungen}

Die Rahmenbedingungen sollten einen möglichst realistischen Alkoholkonsum ermöglichen, der ausschließlich oral und nicht intravenös stattfindet. Die Aufnahmemenge, die Alkoholart und die Trinkgeschwindigkeit sollte von jedem Probanden individuell bestimmt werden können. Es sollte keinerlei Trinkzwang geben und für die gleichzeitige Nahrungsaufnahme in beliebiger Menge war gesorgt.

Ferner sollten auch die praktischen Radfahrversuche möglichst realistisch und damit möglichst nah an die Bedingungen des Straßenverkehrs angelehnt sein. So fanden die Radfahrversuche auf üblichem Straßenbelag (Asphalt und Verbundpflastersteine) bei verschiedenen Wetter- und Lichtbedingungen statt. Die getroffenen Sicherheitsvorkehrungen (s. Punkt 2.2) erlaubten den Probanden ein weitestgehend unbeeinflusstes Fahrradfahren.

\subsubsection{Pilotversuch und Versuchstage}

Nach Durchführung eines ca. 12-stündigen Pilotversuches mit neun Probanden erfolgten an fünf weiteren Terminen Fahrversuche von je ca. 24 Stunden Dauer mit Probandenzahlen zwischen 13 und 18 (Zeit- und Ablaufplan s. Abb. 1; Details zu den Probanden s. Punkt 4). Alle angemeldeten Teilnehmer des Projektes konnten ihre Teilnahme jederzeit und ohne Angabe von Gründen widerrufen, ohne dass dies für die Probanden mit Nachteilen verbunden war, was die Planungssicherheit der Versuche jedoch teilweise maßgeblich beeinträchtigte.

\subsubsection{Alkoholkonsum}

Der Alkoholkonsum erfolgte in geselligem Rahmen auf dem nicht-öffentlichen Testgelände nach Ermittlung der (nüchternen) Ausgangswerte (Auffälligkeiten im Rahmen der ärztlichen Untersuchung, Fahrleistung, Sehleistung). Die eigentliche Alkoholaufnahme begann um die Mittagszeit. Um 23:00 Uhr wurde der Ausschank alkoholhaltiger Getränke gestoppt, um einen weitgehenden Alkoholabbau mit Beendigung des Versuchstages (am folgenden Vormittag) zu gewährleisten. Zwar konnten die Probanden Art und Menge der zugeführten Getränke frei wählen, 
die konsumierten Getränke wurden jedoch mit Uhrzeit des Getränkempfangs auf einem „Laufzettel“ (s. Abb. 2 u. 3) vermerkt.

\section{Zeit- und Ablaufplan der Versuche}

11:00 Uhr Treffpunkt im Institut für Rechtsmedizin, am Universitätsklinikum Düsseldorf

ab 11:15 Uhr Abfahrt nach Neuss mit einem Bus

ab 11:30 Uhr Einweisung in den Versuchsablauf durch den Projektleiter

ab 11:40 Uhr Nummernvergabe, Einverständniserklärungen, Inspektion der ärztlichen Gesundheitszeugnisse, Schweigepflichterklärung

ab 12:00 Uhr Bestimmung der Ausgangswerte:

Blutentnahme incl. erster ärztlicher Untersuchung, Urinprobe, Anprobe der Schutzausrüstung, Probefahrten mit anschließendem 1. Fahrversuch, erste augenärztliche Untersuchung.

13:00 Uhr Freigabe der Bar (für Probanden mit absolviertem Nüchternfahrversuch) und des Büffets

ab 13:15 Uhr Regelmäßige Alco-Tests bei den Probanden - bei Erreichen bestimmter Promillewerte $(0,3 ; 0,6 ; 0,9 ; 1,2 ; 1,5)$ war folgende Reihenfolge vorgeschrieben:

Station 1) Dräger Alcotest $9510 \mathrm{DE}$

Station 2) Blutentnahme

Station 3) Fahrversuch

Station 4) Augenarzt

23:00 Uhr Ende des Alkoholausschanks

\section{Folgetag :}

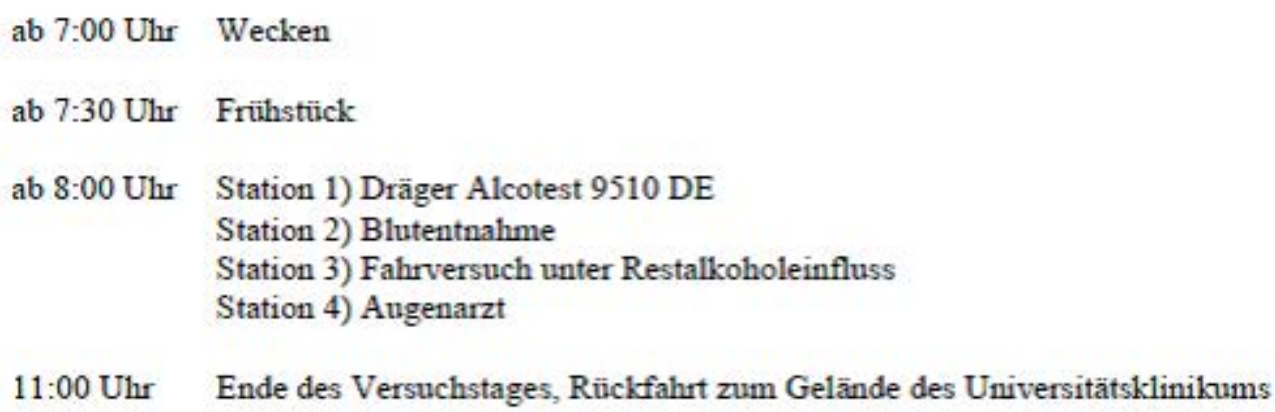

11:00 Uhr Ende des Versuchstages, Rückfahrt zum Gelände des Universitätsklinikums

\section{Abbildung 1:}

\section{Zeit- und Ablaufplan}




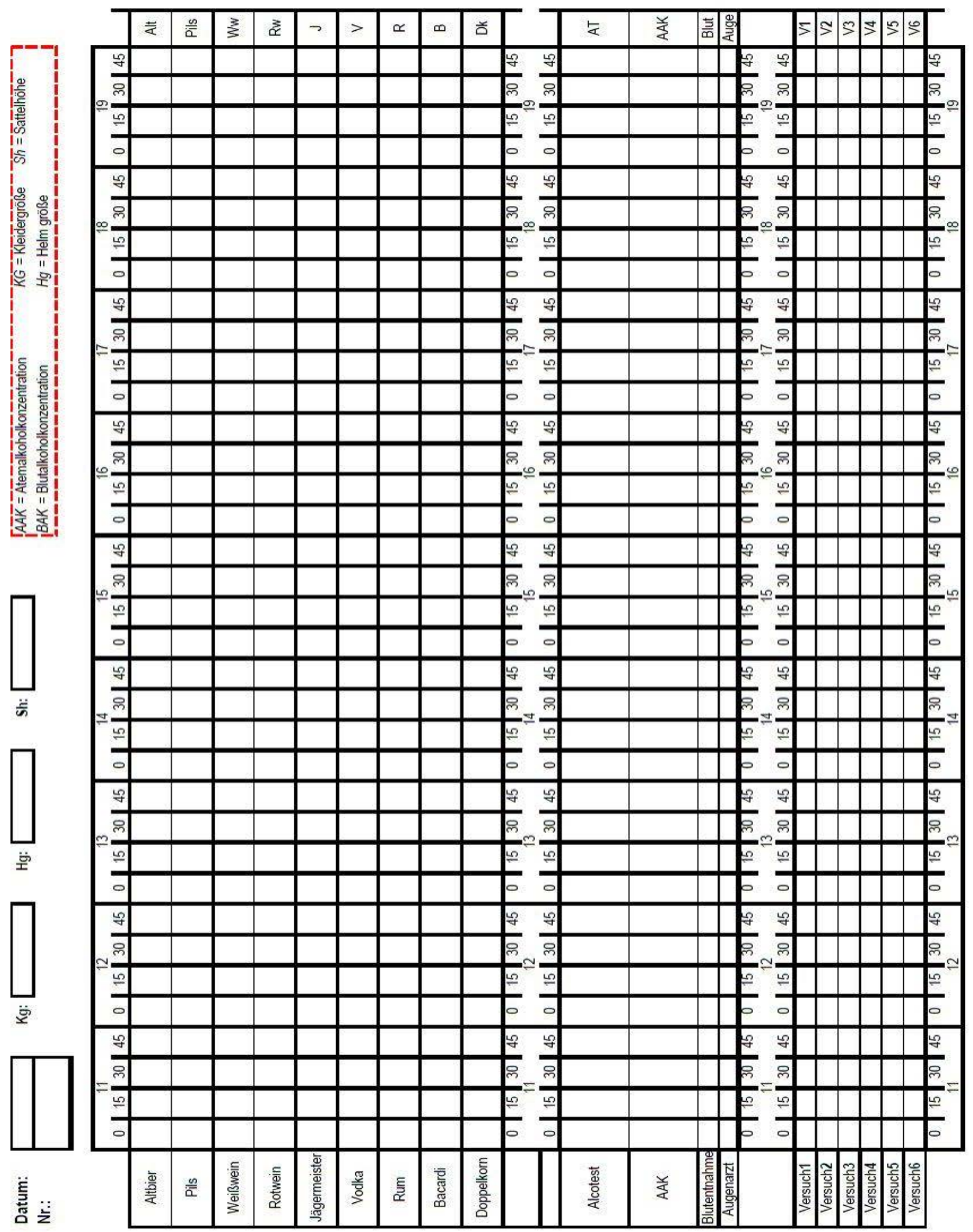

Abbildung 2:

Probandendatenblatt („Laufzettel“) Vorderseite 


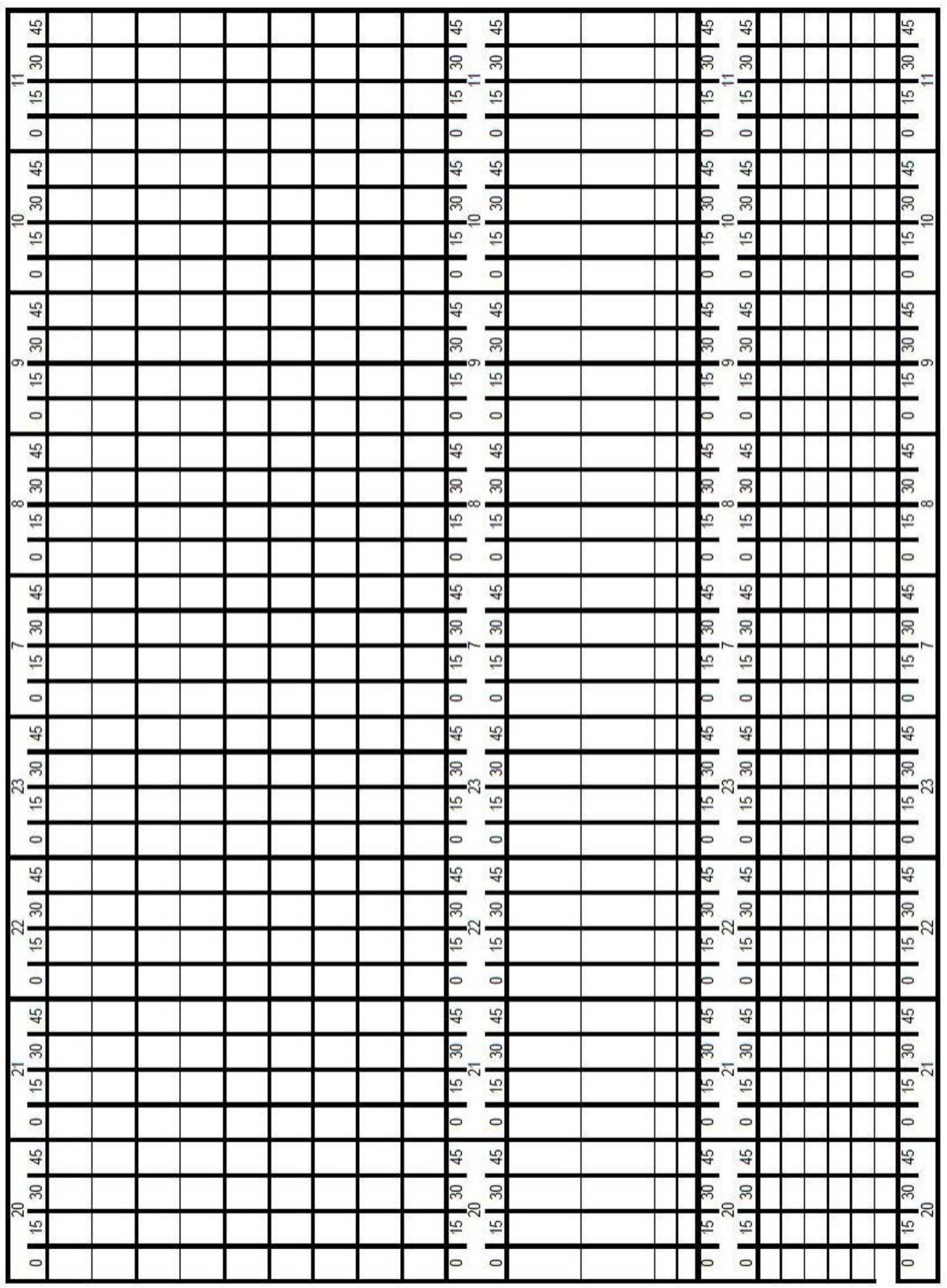

Abbildung 3:

Probandendatenblatt („Laufzettel“) Rückseite 


\subsubsection{Atemalkoholmessungen}

Während die Probanden in individueller Geschwindigkeit Alkohol konsumierten, erfolgten regelmäßige Atemalkoholmessungen (Alcotest mittels Dräger 6510). Bei ungefährem Erreichen des jeweiligen Zielwertes der BAK von 0,3\%o, 0,6\%o, 0,9\%, 1,2\%o oder 1,5\%o erfolgte eine Kontrolle der Atemalkoholkonzentration mittels des Gerätes Dräger Alcotest 9510 DE, ferner am Folgemorgen zur Feststellung etwaigen „Restalkohols“.

\subsubsection{Erreichen eines BAK-Zielwertes}

Wenn aufgrund der festgestellten Atemalkoholkonzentration (Dräger Alcotest 9510 DE) davon auszugehen war, dass einer der vorgenannten BAK-Zielwerte erreicht worden war, wurden Testungen der körperlichen und geistigen Leistungsfähigkeiten vorgenommen und ein praktischer Fahrversuch absolviert. Die Untersuchungen in alkoholisiertem Zustand fanden dabei in immer gleicher Reihenfolge statt: Ärztliche Untersuchung, venöse Blutentnahme zur Bestimmung der BAK, praktischer Fahrversuch, augenärztliche Untersuchung). Im Anschluss an eine Testreihe konnte weiterhin Alkohol konsumiert werden, bis aufgrund der Atemalkoholmessergebnisse erneut davon auszugehen war, dass der nächste BAK-Zielwert erreicht worden ist.

\subsubsection{Untersuchung am Folgemorgen}

Zur Untersuchung des Einflusses von Restalkohol bzw. zur Untersuchung der Leistungsfähigkeit im nüchternen Zustand (jedoch nach erheblichem Alkoholkonsum) erfolgte eine zusätzliche Testreihe am Folgemorgen.

\subsubsection{Abbruchkriterien}

Wenn ein Proband nicht bis zu einer BAK von etwa 1,5\%o trinken konnte bzw. wollte, wurde dessen individueller Endpunkt festgehalten. Wenn ein Proband alkoholbedingte Defizite zeigte, die mit dem sicheren Führen eines Fahrrades nicht mehr vereinbar waren, z.B. erhebliche Anfahrschwierigkeiten, akutes Erbrechen, stark schwankender Gang, unterblieben weitere Fahrversuche in diesem Zustand, um kein unnötiges Verletzungsrisiko einzugehen. 


\subsection{Maßnahmen zur Verhinderung von Unfällen und Verletzungen}

Um Unfälle mit Verletzungen der Versuchsteilnehmer weitestgehend auszuschließen, wurden die Versuche auf einem abgesperrten Gelände mit geeigneten Sicherheitsrädern (Lenkerpolsterung, ohne oberes Rahmenrohr, federnde 8 Zoll große Stützräder neben beiden Hinterreifen, die erst im Falle eines Kippvorganges aufsetzten) aus dem Rehabilitationswesen durchgeführt. Während aller Fahrversuche trugen die Probanden einen leichten Motorradschutzanzug mit integrierten Knie- und Ellenbogenprotektoren. Für den Transport der Versuchsteilnehmer zwischen dem Treffpunkt beim Universitätsklinikum Düsseldorf und dem Versuchsgelände wurde ein Shuttle-Service eingerichtet, wobei alle Versuchsteilnehmer angewiesen waren, bereits mit öffentlichen Verkehrsmitteln zum Klinikumsgelände anzureisen. Für jeweils maximal drei Probanden stand während der Versuche jeweils mindestens eine Betreuungsperson zur Verfügung. Neben mindestens einem ganzzeitig anwesenden Arzt wurden die Versuche von jeweils einem Rettungssanitäter und einem Rettungsassistenten inkl. Rettungsfahrzeug begleitet. Unmittelbar vor Entlassung der Probanden vom Testgelände erfolgte eine letzte Atemalkoholkontrolle. Probanden mit einer Atemalkoholkonzentration von weniger als $0,15 \mathrm{mg} / \mathrm{l}$ wurden aus der Untersuchung entlassen. Probanden mit einer Atemalkoholkonzentration von $0,15 \mathrm{mg} / \mathrm{l}$ oder mehr wurden zur eigenen Wohnanschrift gefahren.

Die Versuchsanordnung wurde vor Versuchsbeginn von der Ethikkommission der Heinrich-HeineUniversität Düsseldorf mit Schreiben vom 18.04.2013 genehmigt (Studiennummer 4086).

\subsection{Praktische Anordnung der Versuche}

Grundlagen der Versuche (Skizze des Parcours in Abb. 4) waren die vier von Schewe et al. [1, 2] durchgeführten Experimente:

- Geradeausfahrt auf einer sich verjüngenden Fahrspur: Die $45 \mathrm{~m}$ lange, gerade verlaufende, ebene Fahrstrecke verjüngte sich von $2 \mathrm{~m}$ Breite zu Fahrtbeginn nach $10 \mathrm{~m}$ Fahrtstrecke auf 1,50 m, nach weiteren $10 \mathrm{~m}$ Fahrtstrecke auf $1 \mathrm{~m}$ und verlief dann weitere $10 \mathrm{~m}$ konisch in eine $15 \mathrm{~m}$ lange, 0,30 m schmale Fahrspur über (Abb. 4, Markierung a und Abb. 5).

- Durchfahren seitenversetzter Tore: Nach der Geradeausfahrt waren insgesamt sechs Tore zu durchfahren, wobei der innere Abstand zwischen den Slalomstangen 1,50 m betrug (Abb. 4, Markierung b und Abb. 6). 
- Slalomfahrt durch in Linie aufgestellte Kegel mit kleiner werdendem Abstand: Es waren insgesamt zehn in Reihe aufgestellte Kegel zu umfahren, die in kleiner werdendem Abstand aufgestellt waren. Zunächst drei Kegel in Abständen von je $4 \mathrm{~m}$, dann weitere drei Kegel im Abstand von je $3 \mathrm{~m}$, dann zwei Kegel mit $2 \mathrm{~m}$ Entfernung und am Ende ein Kegel in einer Distanz von 1,50 m (Abb. 4, Markierung c und Abb. 6).

- Dreimaliges Durchfahren eines Kreises mit Innenradius von 3 m und Außenradius von $4 \mathrm{~m}$ im Uhrzeigersinn (Abb. 4, Markierung g).

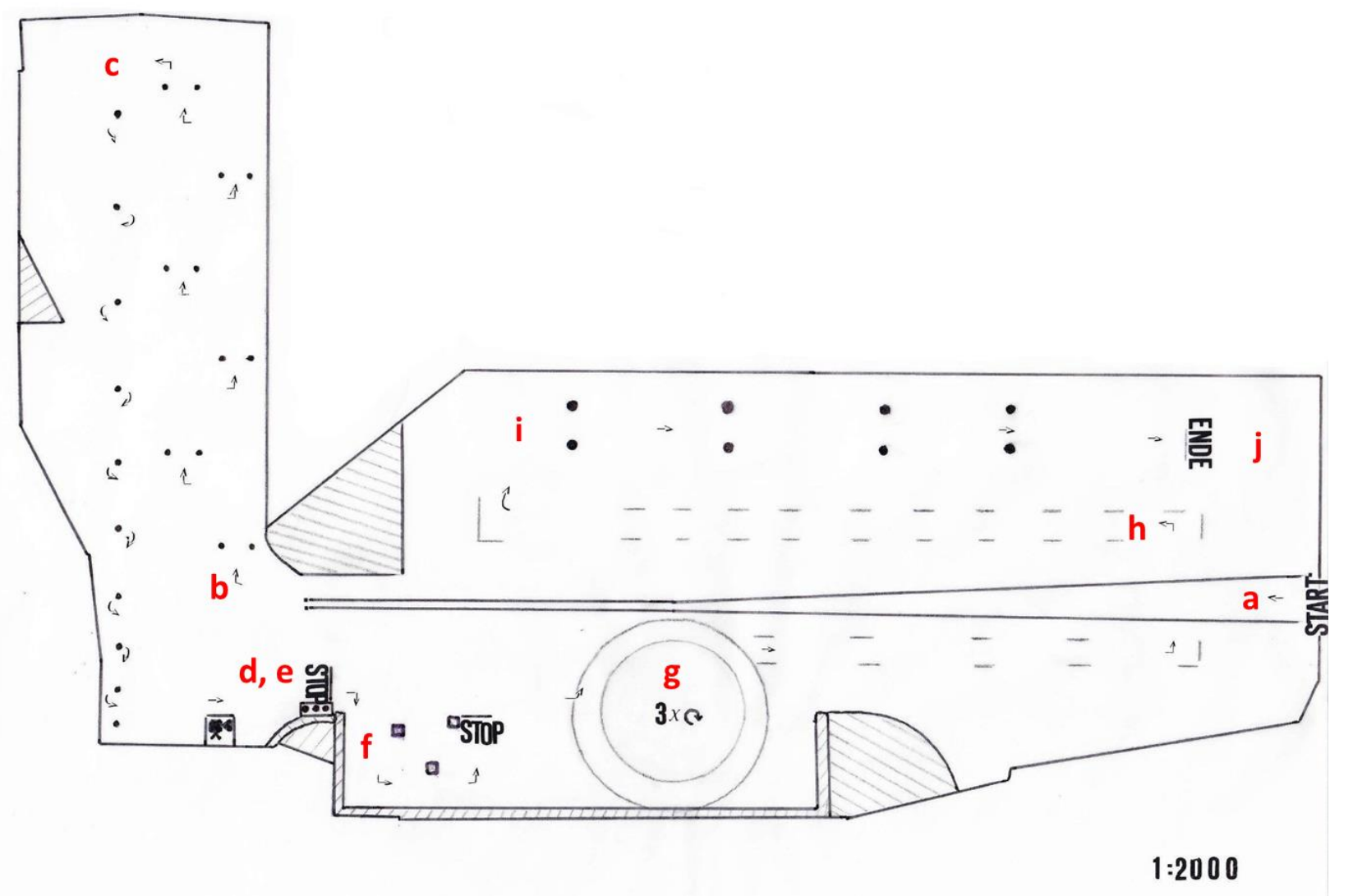

\section{Abbildung 4:}

Skizze des Parcours 


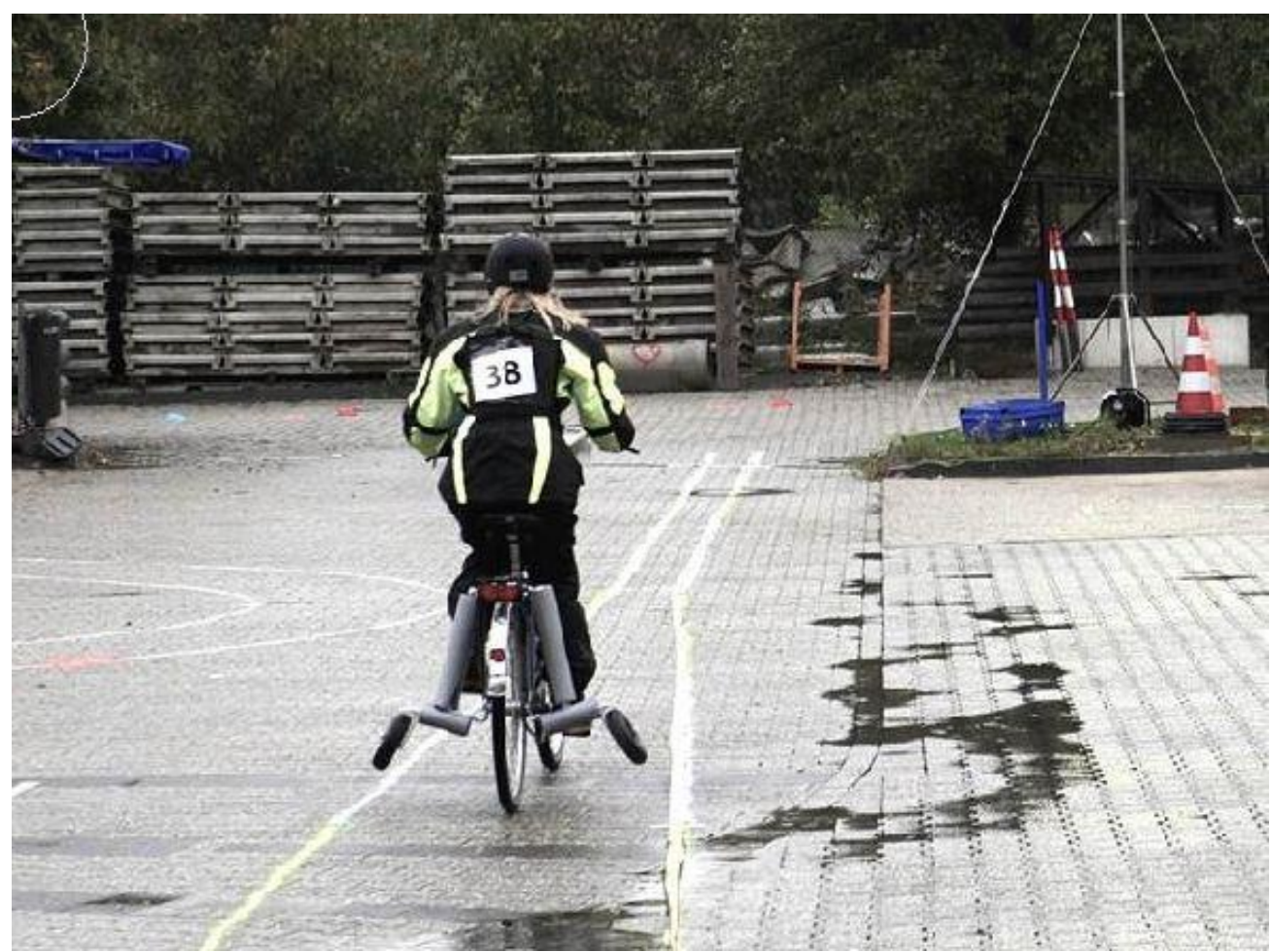

\section{Abbildung 5:}

Geradeausfahrt auf einer sich verjüngenden Fahrspur

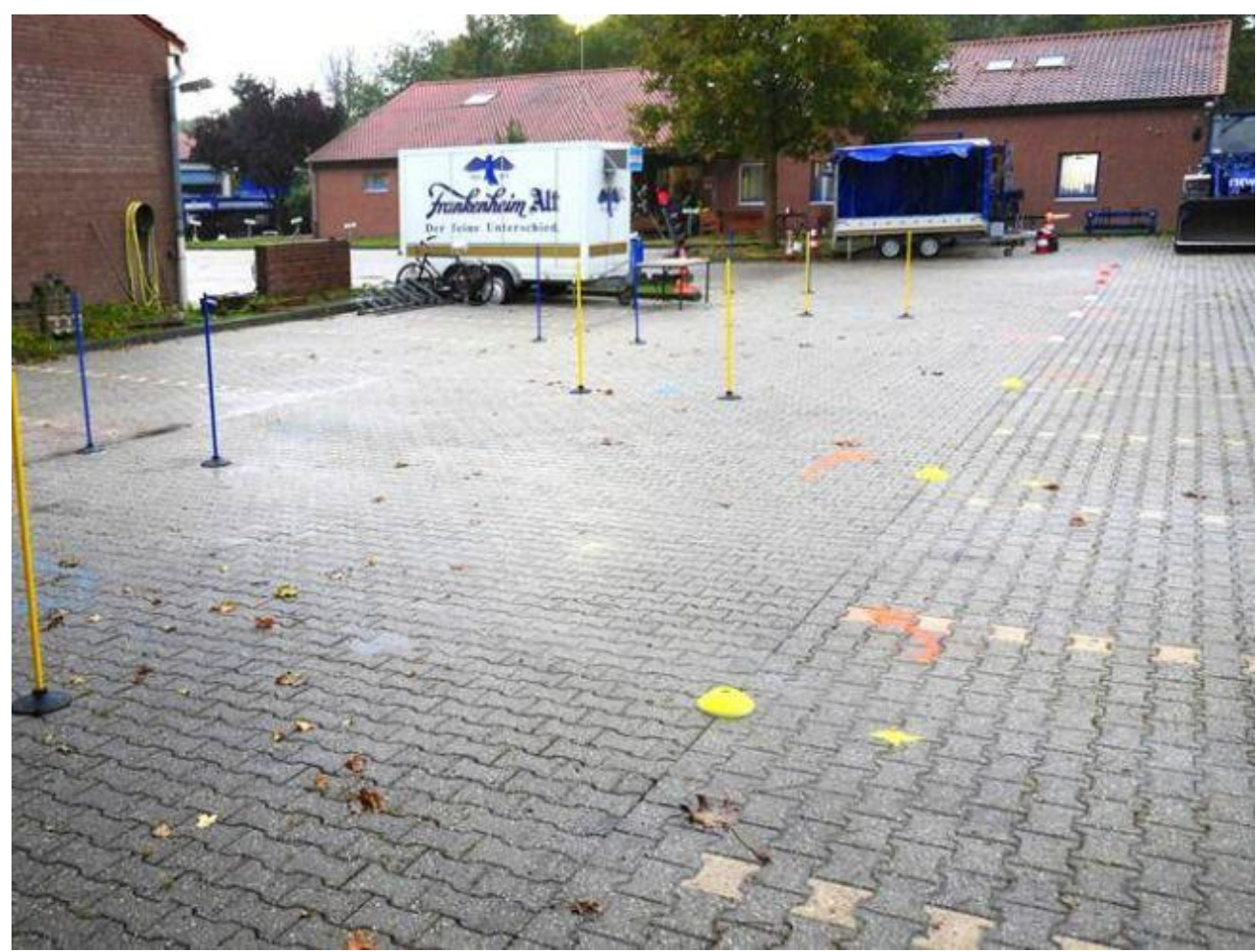

Abbildung 6:

Durchfahren seitenversetzter Tore (linke Bildhälfte) und Slalomfahrt durch in Linie aufgestellte Kegel mit zunehmend geringerem Abstand (rechte Bildhälfte) 
Zusätzlich wurden die von Schewe übernommenen Punkte durch weitere Prüfstationen ergänzt:

- Es erfolgte ein Reaktionstest an einer manuell einstellbaren Ampelanlage (Abb. 4, Markierung d und Abb. 7).

- Im Bereich der Ampel wurde auf einem Leuchtdisplay ein Wort eingespielt, das zwischen den Fahrversuchen regelmäßig variierte (Abb. 4, Markierung e und Abb. 7). Dieses Wort wurde nach Beendigung des Fahrversuchs abgefragt und notiert (Abb. 4, Markierung j).

- Unmittelbar nach dem Anhaltevorgang an der manuell verstellbaren Ampel musste nach dem Umfahren von Markierungshütchen an einer STOP-Linie angehalten werden (Abb. 4, Markierung f).

- Es wurde die Reaktion auf komplexe, unvorhersehbare Situationen beobachtet (Abb. 4, Markierung h), wie z.B. ein in den Fahrweg rollender Ball, das Blenden mit einer Taschenlampe, Zwischenrufe oder Blockade bzw. Einengung der Fahrspur.

- Zuletzt musste eine 20 m lange Gasse durchfahren werden, die von Plastiktonnen begrenzt wurde. Diese Plastiktonnen standen in Abständen von $5 \mathrm{~m}$ in Reihe zueinander und wiesen Innenabstände (der Gassenbreite entsprechend) von 1,35 m auf (Abb. 4, Markierung i).

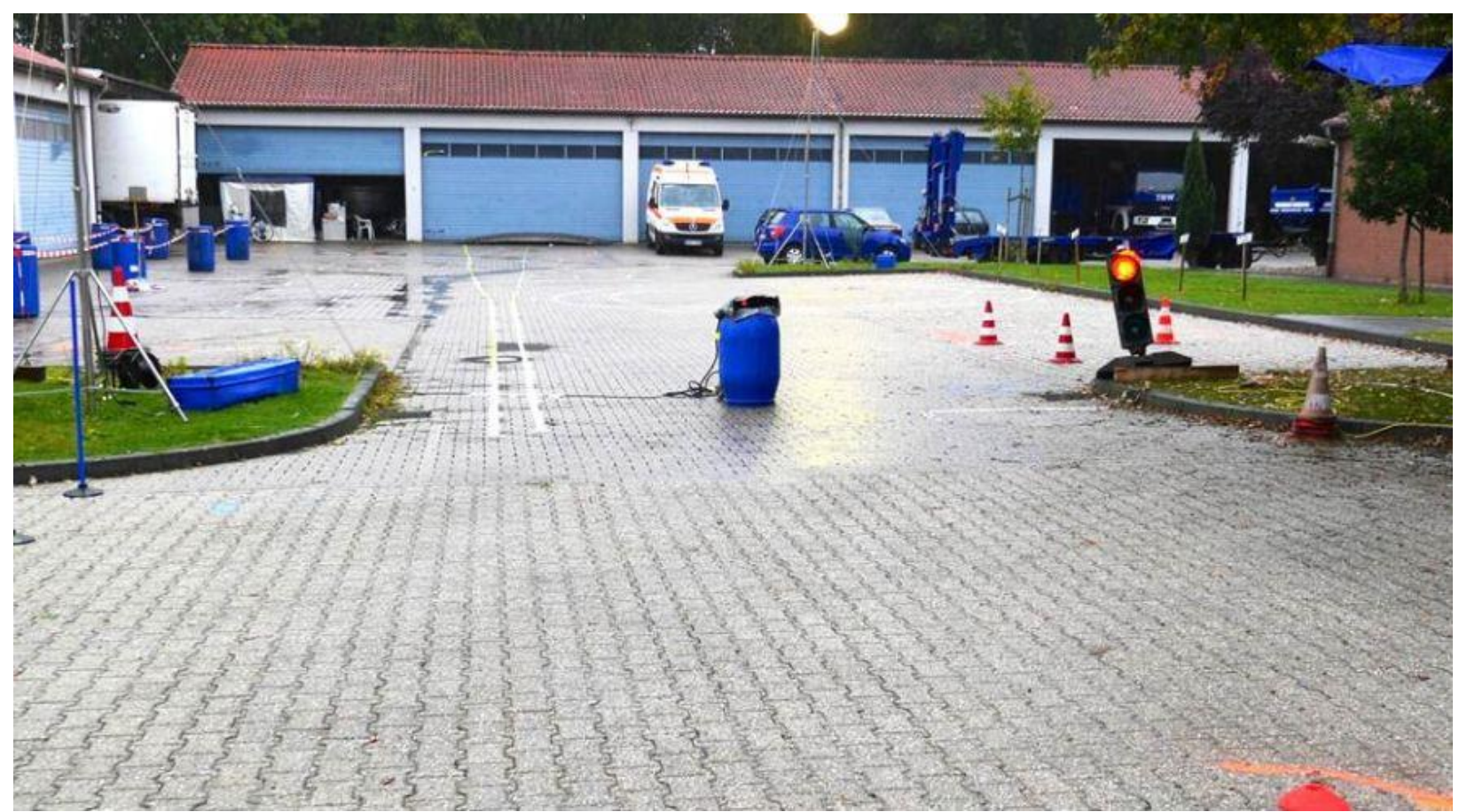

\section{Abbildung 7:}

Ampel (rechts) mit Leuchtdisplay (auf blauer Tonne angebracht) 
Die Versuche wurden mittels zweier Videokameras aufgezeichnet (Kamera 1: mitfahrend, Montage am Fahrradlenker; Kamera 2: stationär, auf Stativ montierte Kamera an zentralem Punkt für Übersichtsaufnahme).

\section{$2.4 \quad$ Versicherung}

Für alle Probanden wurden eine Haftpflichtversicherung sowie eine verschuldensunabhängige Probandenversicherung abgeschlossen. Alle Teilnehmer wurden über die Versicherung und ihre Obliegenheiten vor Versuchsbeginn aufgeklärt.

\subsection{Drogenscreening}

Vor Testbeginn gaben die Probanden eine Urinprobe zur Untersuchung auf berauschende Mittel (immunchemisches Screening) ab, um mögliche Wechselwirkungen mit Alkohol aufzudecken. Die Urinproben wurden im Anschluss mittels LC/MS-Untersuchungen aufgearbeitet (Flüssigkeitschromatographie mit Massenspektrometrie-Kopplung). Bei positiven Drogenbefunden erfolgte eine Wirkstoffbestimmung im Blut mittels GC/MS (Gaschromatographie mit Massenspektrometrie-Kopplung).

\subsection{Datenschutz}

Die Wahrung sämtlicher datenschutzrechtlicher Bestimmungen wurde gewährleistet. Den entnommenen Blut- und Urinproben wurden mittels einer Pseudonymisierungsliste Nummern zugeordnet, wobei die Aufbewahrung dieser Liste an einem getrennten Ort erfolgte. Die Ergebnisse der Untersuchungen wurden ausschließlich durch unmittelbar an dem Projekt beteiligte sachverständige Wissenschaftler analysiert, die der Schweigepflicht unterlagen bzw. unterliegen. 


\subsection{Fahrräder}

Für die Versuche wurden Spezialfahrräder aus dem Behinderten- bzw. Reha-Bereich (26“Laufräder, Rücktrittbremse, Vorderradtrommelbremse, Feststellbremsgriff, Lichtanlage, Gel- Sattel, Rahmen ohne obere Stange) beschafft, die eine Lenkerpolsterung sowie federgedämpfte Stützräder neben beiden Hinterreifen aufwiesen (s. Abb. 8). Die Stützräder von 8“ Durchmesser befanden sich im Abstand von jeweils etwa $35 \mathrm{~cm}$ zum Laufrad, hatten jedoch nur im Falle eines Kippvorganges oder bei sehr zügiger Kreisfahrt Bodenkontakt. Bei Geradeausfahrt befanden sich die Stützräder jeweils $17 \mathrm{~cm}$ vom Boden entfernt.

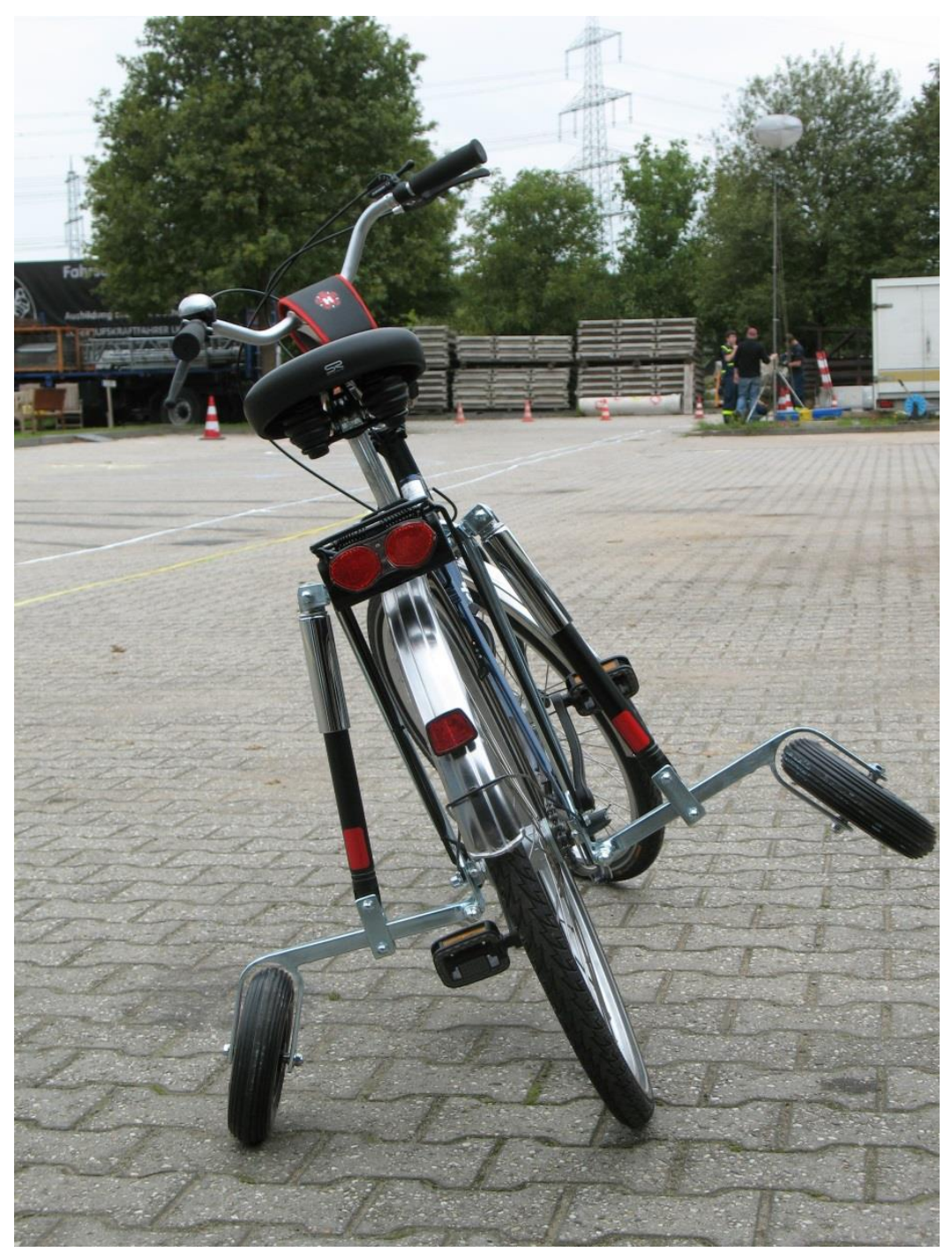

\section{Abbildung 8:}

Eines des Sicherheitsfahrräder, mit denen die Fahrversuche durchgeführt wurden 


\subsection{Schutzausrüstung}

Zur Schutzausrüstung gehörten unterschiedliche Größen von individuell verstellbaren Fahrradschutzhelmen nach Europäischer Norm EN 1078 sowie Motorradschutzanzüge der Marke „Hein Gericke“ in textiler Ausführung, die mit Schulter-, Ellbogen-, Rücken-, Hüft- und Knieprotektoren ausgestattet waren (s. Abb. 9).

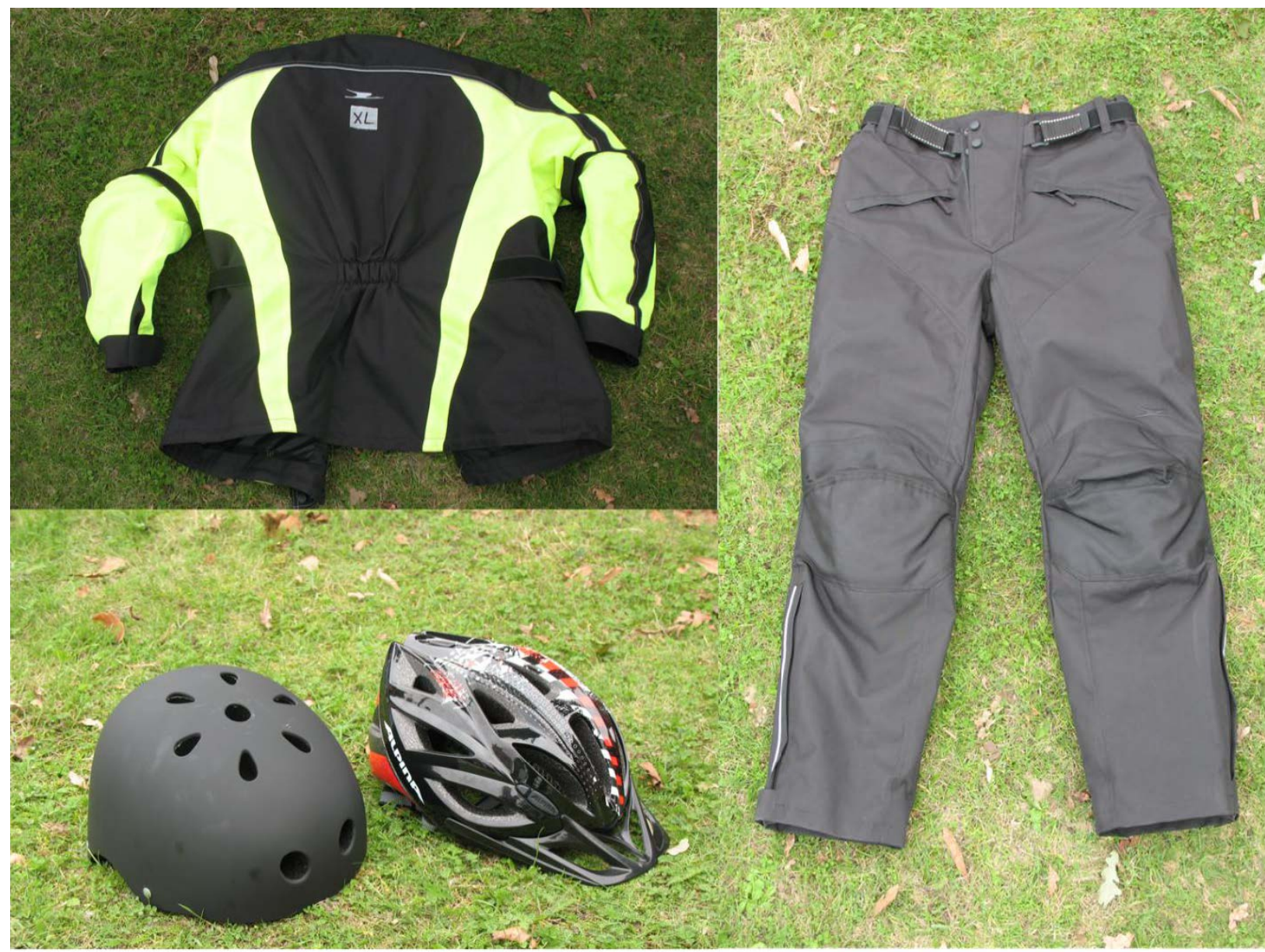

\section{Abbildung 9:}

Motorradschutzausrüstung, die während der Fahrversuche getragen werden musste

\subsection{Atemalkoholtestgerät Dräger Alcotest 9510 DE}

Für die Versuche wurde das aktuelle beweissichere Atemalkoholmessgerät Dräger Alcotest 9510 DE mit der auch von polizeilicher Seite benutzten Software verwendet. Das Gerät erfüllt die Anforderungen der DIN VDE 0405 und ist von der Physikalisch-Technischen Bundesanstalt zugelassen. Die Bauartzulassung wurde kurz vor dem Experiment im August 2013 erteilt. Vor der Messung wurden alle relevanten Daten mittels Touchscreen in das Gerät eingegeben. Das Gerät verfügt über einen internen Thermopapier-Drucker, so dass direkt im Anschluss an die Alkoholmessungen alle relevanten Informationen (Datum, Uhrzeit, Vorfallszeitpunkt, 
Messergebnis, Atemvolumen und -zeit, Probandenname bzw. -nummer, Geschlecht, Geburtsdatum und Name des Bedieners) ausgedruckt werden konnten.

\section{4 Ärztliche Untersuchung im Rahmen der Blutentnahmen}

Vor jedem Fahrversuch fand neben einer Blutentnahme auch eine ärztliche Untersuchung zur Feststellung über die Auswirkungen der Alkoholaufnahmen statt, wie sie auch regulär bei Verdacht auf Führen eines Fahrzeugs unter Alkoholeinfluss vorgenommen wird. Dabei wurden folgende geistige und körperliche Fähigkeiten untersucht (s. Abb. 10):

- Finger-Finger-Prüfung,

- Finger-Nasen-Prüfung,

- Gang geradeaus,

- plötzliche Kehrtwendung (nach vorherigem Gehen),

- Pupillenweite,

- Bindehautveränderungen,

- Bewusstsein,

- Sprache,

- Denkablauf,

- Befinden,

- Stimmung,

- Verhalten,

- äußerliche Erscheinung der Alkoholauswirkung.

Bei einem Teil der Probanden wurde zusätzlich der Drehnystagmus überprüft. Die Prüfung des Drehnystagmus erfolgte, indem der Proband bei geöffneten Augen innerhalb von 10 Sekunden fünf Mal um die eigene Vertikalachse gedreht wurde. Anschließend wurde die Dauer des Augenzuckens bei vor die Augen gehaltenem Zeigefinger gemessen.

\subsection{Augenärztliche Untersuchungen}

Nach jedem Fahrversuch erfolgten augenärztliche Untersuchungen hinsichtlich der inneren und äußeren Fusionsbreite (Angabe in Dioptrien). Mit diesem Test wurde überprüft, inwieweit der Proband in der Lage war, die Bilder, die die beiden Augen liefern, im Sehzentrum zu einem einzigen Bild zusammen zu führen: Zur Ermittlung der Fusionsbreite wurden den Probanden in verschiedenen Alkoholisierungsstadien Prismen vor die Augen gehalten, während diese einen 
etwa 1,5 m entfernten Punkt an der Wand fixieren mussten. Ermittelt wurde der Wert, ab dem die Probanden nicht mehr in der Lage waren, den Punkt als einen einzigen Punkt wahrzunehmen (Sehen von Doppelbildern). Ferner wurde ein sogenannter Swinging-Test durchgeführt, bei dem die Probanden den sich abwechselnd nach links und rechts bewegenden Zeigefinger des Untersuchers mit ihrem eigenen Zeigefinger insgesamt zehn Mal berühren mussten. Die hierfür benötigte Zeit wurde gestoppt. Drittens wurde die Zeit gemessen, die zum Vorlesen eines definierten Buchtextabschnittes von etwa 50 Wörtern bei den verschiedenen Alkoholisierungsgraden benötigt wurde.

Feststellungen über die Auswirkungen der Alkoholaufnahme

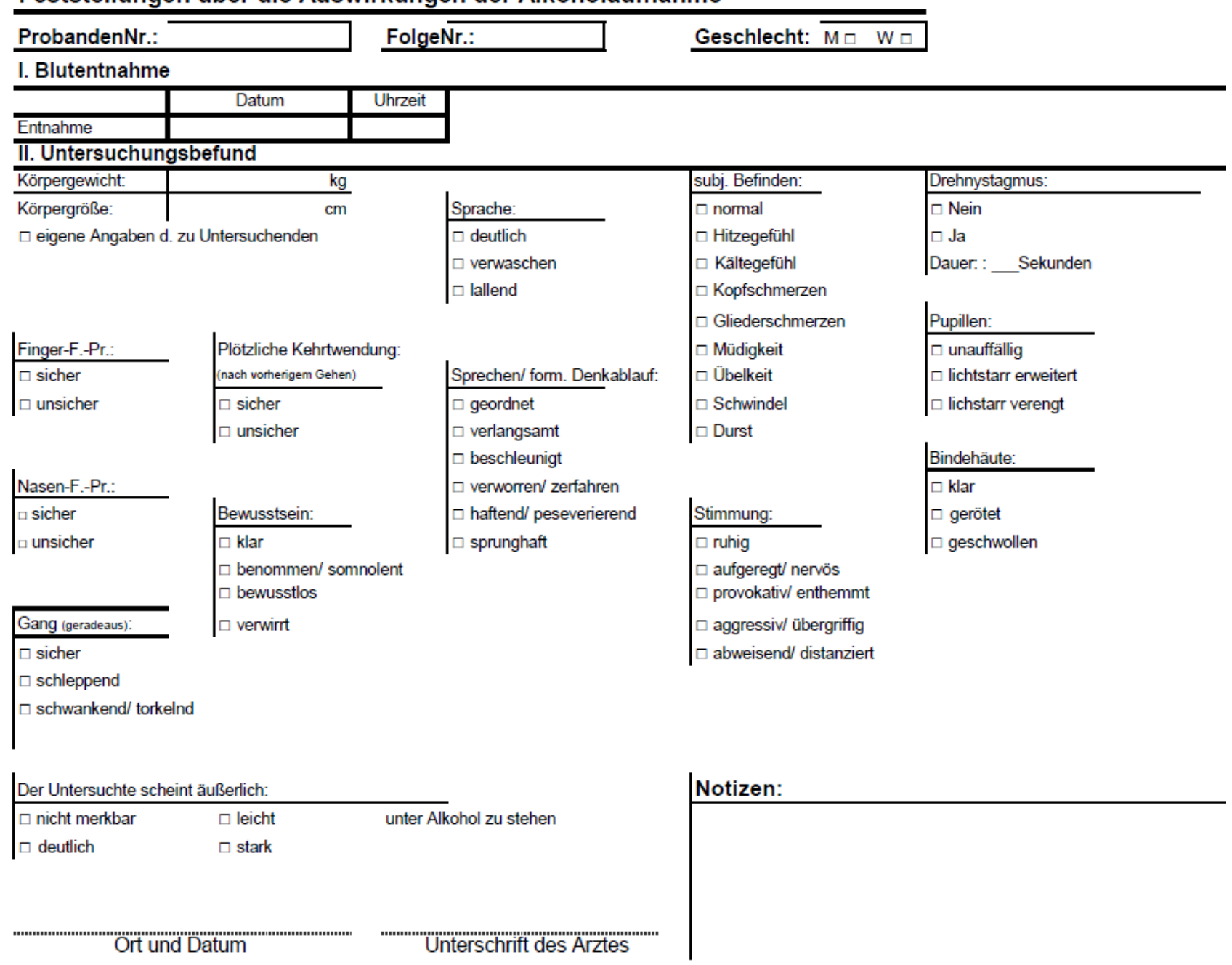

\section{Abbildung 10:}

Formular für den ärztlichen Bericht zur Blutentnahme 


\subsection{Alkoholika}

Der Alkoholkonsum der Probanden erfolgte zu beliebigen Zeitpunkten nach freier Wahl aus der folgenden Getränkeliste (Mischungen mit analkoholischen Getränken waren jederzeit möglich):

- Altbier: Frankenheim

$50 \mathrm{cl}, 4,8$ Vol.- $\%, 200 \mathrm{ml}$ Glas

- Pils: Bitburger

$50 \mathrm{cl}, 4,8$ Vol.- $\%, 200 \mathrm{ml}$ Glas

- Rotwein I: „Acolon“, 2009 Affaltracher Salzberg, Württemberg

$75 \mathrm{cl}, 11,5$ Vol.- $\%, 200 \mathrm{ml}$ Glas

oder

Rotwein II: „, Lemberger“, 2010 Affaltracher Salzberg, Württemberg

$75 \mathrm{cl}, 12,0$ Vol.- $\%, 200 \mathrm{ml}$ Glas

- Weißwein I: „, Jechtinger“, 2010 Grauer Burgunder, Baden

$75 \mathrm{cl}, 12,5$ Vol.-\%, $200 \mathrm{ml}$ Glas

oder

Weißwein II: „Oberbergener Bassgeige“, 2012 Baden, Grauer Burgunder, Baden

$75 \mathrm{cl}, 12,5$ Vol.- $\%, 200 \mathrm{ml}$ Glas

- Kornbrand: Doppelkorn, Braun goldene Meile

$70 \mathrm{cl}, 38 \mathrm{Vol} .-\%, 20 \mathrm{ml}$ Glas

- Kornbrand: Fürst Bismarck

70cl, 38 Vol.-\%, $20 \mathrm{ml}$ Glas

- Kräuterlikör: Jägermeister

$70 \mathrm{cl}, 35 \mathrm{Vol} .-\%, 20 \mathrm{ml}$ Glas

- Rum: Bacardi

70cl, 37,5 Vol.-\%, $20 \mathrm{ml}$ Glas

- Rum: Captain Morgan

70cl, 35 Vol.-\%, 20 ml Glas 
- Wodka: Gorbatschow

70cl, 37,5 Vol.-\%, $20 \mathrm{ml}$ Glas

\subsection{Videokameras}

Alle Fahrversuche wurden mit zwei Videokameras aufgezeichnet. Zentral an die Lenkerstange wurde eine „GoPro HERO 3 Black Edition“-Kamera montiert. Eine zweite, fest installierte Sony HXR-NX5e-Kamera wurde von einem eigenen Kameramann bedient.

\section{8 Übernachtung}

Die Übernachtung erfolgte auf dem Testgelände in einer Gemeinschaftsunterkunft auf Feldbetten. Schlafsäcke sowie Kopfkissen wurden aus hygienischen Gründen von den Probanden selbst mitgebracht. Nach dem Zubettgehen des ersten Probanden erfolgten durch die Versuchsbetreuer regelmäßige Kontrollgänge.

\subsection{Auswertung}

Für die Auswertung der im Rahmen der ärztlichen Untersuchungen und während der praktischen Fahrversuche feststellbaren Auffälligkeiten erfolgte die Vergabe von Punktwerten. Verkehrsmedizinisch relevantere Auffälligkeiten erhielten dabei höhere Punktwerte als solche mit geringerer Relevanz. Die vergebenen Punktwerte sind unter Punkt 5 aufgeführt.

Die statistische Auswertung wurde durch Prof. Dr. Holger Schwender, Institut für Mathematik der Heinrich-Heine-Universität Düsseldorf, vorgenommen. Abbildungen, die Regressionslinien (rot) enthalten, sind so zu verstehen, dass die schwarzen Linien die Messergebnisse der einzelnen Probanden miteinander verbinden. Jeder schwarze Punkt stellt dabei ein Messergebnis dar. Abbildungen, die Box-Plots enthalten, sind so zu verstehen, dass die Box $50 \%$ der Messergebnisse enthält; die Satelliten nach oben bzw. unten spiegeln jeweils $25 \%$ der Messergebnisse wider; Kreise zeigen Ausreißer an. 


\section{$4 \quad$ PROBANDEN}

Jeder Proband durfte nur an einem der fünf Versuchstage teilnehmen; eine zusätzliche Teilnahme am Pilotversuch war jedoch gestattet. Jeder Proband musste vor Versuchsbeginn ein zeitnah erstelltes ärztliches Gesundheitszeugnis vorlegen, aus dem hervorging, dass keine Hinweise auf eine Leberfunktionsstörung oder neurologische/ psychiatrische Erkrankungen vorliegen. Wir wählten die folgenden Ein- bzw. Ausschlusskriterien:

\section{Einschlusskriterien für Probanden}

- Alter zwischen 18 und 53 Jahren.

- Vorliegen einer unterschriebenen Einwilligungserklärung.

- Vorhandene Fähigkeit, ein Fahrrad zu führen.

- Vorlage des o.g. ärztlichen Gesundheitszeugnisses.

- Erfahrung im Konsum von Alkohol, d.h. keine Alkoholabstinenz.

- Negatives Drogenscreening des Urins vor Versuchsbeginn. Bei einem positiven Drogenscreening des Urins konnte eine Teilnahme unter Vorbehalt nur erfolgen, wenn bei der Eingangsuntersuchung keine klinische Beeinträchtigung vorlag. Die Blutprobe wurde im Nachgang gezielt auf den Drogenwirkstoff untersucht. Nur, wenn keine relevante Wirkstoffkonzentration vorhanden war, war auch in diesem Fall das Einschlusskriterium erfüllt.

\section{Ausschlusskriterien für Probanden}

- Vorliegen von aktuellen neurologischen oder psychiatrischen Erkrankungen.

- Alkoholabhängigkeit in der Vorgeschichte.

- Leberfunktionsstörungen.

- Schwangerschaft.

- Positives toxikologisches Screening des Urins bzw. des Blutes.

In den Pilotversuch wurden neun Probanden eingeschlossen, in die darauf aufbauenden fünf Versuchstage wurden 74 Probanden (Versuch 1: $N=15$, Versuch 2: $N=13$, Versuch 3: $N=13$, Versuch 4: $\mathrm{N}=18$, Versuch 5: $\mathrm{N}=15$ ) einbezogen. Da alle Tests im Rahmen des Pilotversuchs bereits die gestellten Anforderungen an die Versuchsdurchführung erfüllten, wurden auch diese Ergebnisse in die Auswertung einbezogen. Probanden durften dabei maximal am Pilotversuch und einem der fünf Versuchstage teilnehmen. Bei den insgesamt 83 Testreihen waren die Probanden in 39 Fällen weiblich und in 44 Fällen männlich. Der Altersmedian lag bei 25 Jahren (männlich: 
26 Jahre, weiblich: 23 Jahre). Bezüglich der Geschlechter- und Altersverteilung sei auf Abb. 11 verwiesen.

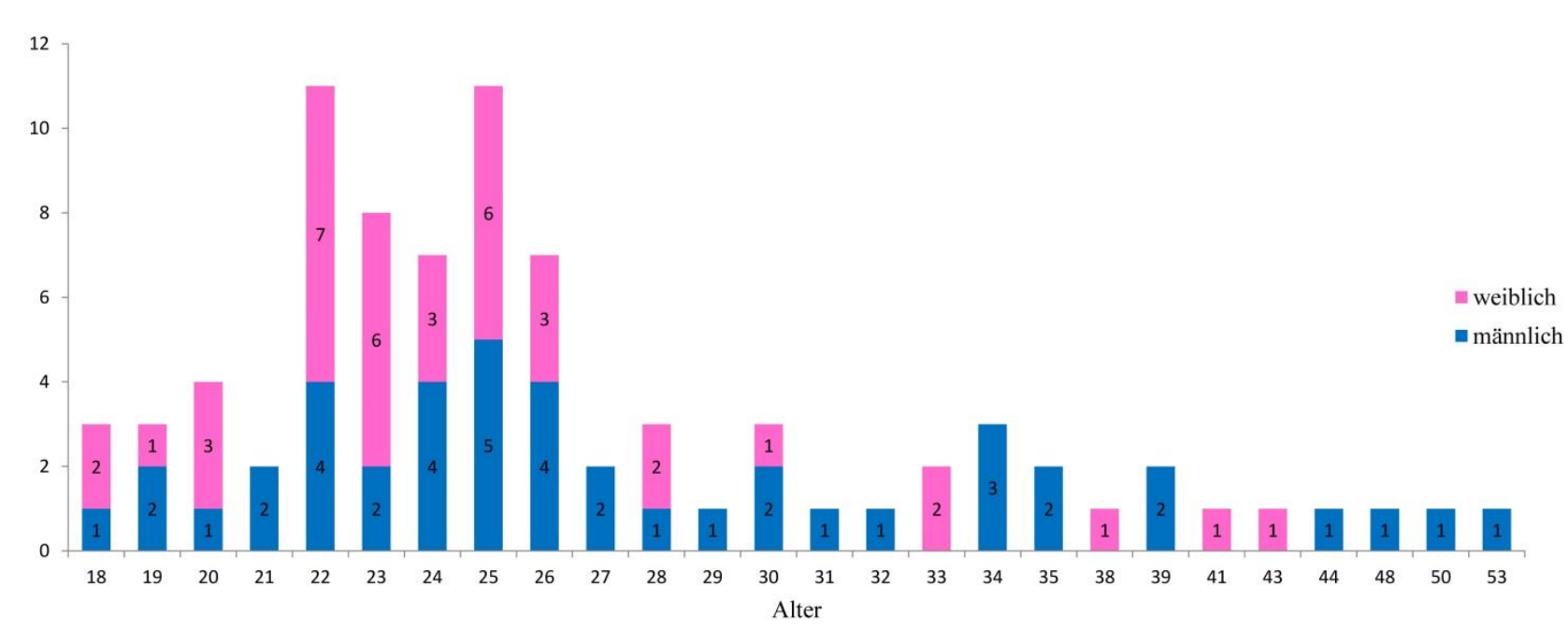

Abbildung 11:

Altersverteilung und Geschlecht der Probanden

\section{$5 \quad$ ERGEBNISSE}

\subsection{Ergebnisse des Drogen- und Arzneimittelscreenings}

Insgesamt gab es drei positive Drogenbefunde (jeweils Cannabis), die im Nachgang mittels GC/MS-Untersuchungen der Blutprobe auf THC (Tetrahydrocannbinol) und THC-Metabolite (11$\mathrm{OH}-\mathrm{THC}$ und THC-COOH) verifiziert werden konnten: Proband Nr. 109 (Pilotversuch), Proband 8 (1. Versuchstag) und Proband 74 (5. Versuchstag). Da es keine Hinweise auf einen Rauschzustand gab, erfolgte die Teilnahme dieser drei Probanden unter Vorbehalt. Die nachgelagerten Blutlaboruntersuchungen ergaben im Einzelnen die im Folgenden aufgeführten THC- bzw. THC-Metaboliten-Werte; weitere illegale psychotrope Substanzen wurden nicht gefunden. Zusätzlich ist der sog. Cannabis Influence Factor (CIF; ohne Einheit) aufgeführt, wobei ein Wert oberhalb von 10 eine akute Beeinflussung anzeigt. Im Rahmen des LC/MS-Screening des Urins wurden ferner das blutdrucksenkende Medikament Metoprolol (Proband 30) sowie die Psychopharmaka Quetiapin und Lamotrigin (jeweils Proband 72) aufgefunden. 
Proband 109; 1. Fahrversuch

THC

11-OH-THC

THC-COOH

CIF

Proband 8; 1. Fahrversuch

THC

11-OH-THC

THC-COOH

CIF
$0,5 \mathrm{ng} / \mathrm{ml}$

$0,1 \mathrm{ng} / \mathrm{ml}$

$3,7 \mathrm{ng} / \mathrm{ml}$

\section{Proband 109; 5. Fahrversuch}

THC nicht sicher nachweisbar

11-OH-THC nicht sicher nachweisbar

THC-COOH $\quad 2,4 \mathrm{ng} / \mathrm{ml}$

CIF
Proband 8; 4. Fahrversuch

THC

$1,2 \mathrm{ng} / \mathrm{ml}$

11-OH-THC

$0,4 \mathrm{ng} / \mathrm{ml}$

THC-COOH

$12 \mathrm{ng} / \mathrm{ml}$

CIF

14
Proband 74; 1. Fahrversuch

THC

11-OH-THC

THC-COOH

CIF

$0,2 \mathrm{ng} / \mathrm{ml}$

$0,2 \mathrm{ng} / \mathrm{ml}$

$4 \mathrm{ng} / \mathrm{ml}$
Proband 74; 4. Fahrversuch

THC

nicht sicher nachweisbar

11-OH-THC

$0 \mathrm{ng} / \mathrm{ml}$

THC-COOH

$2,3 \mathrm{ng} / \mathrm{ml}$

CIF

\subsection{Erreichte Blutalkoholkonzentrationen (BAK)}

Die im Rahmen des ersten Fahrversuchs (Nüchternversuch) ermittelten BAK lagen mit einer Ausnahme bei allen 83 Probanden bei 0,00 \% . Proband Nr.15 wies eine BAK von 0,02 \%o auf, die sich in der nahezu zeitgleich erfolgten Atemalkoholkontrolle (AAK) nicht gezeigt hatte.

Das Trinkverhalten der einzelnen Probanden war sehr unterschiedlich, so dass teilweise der angestrebte BAK-Bereich, in dem ein Fahrversuch erfolgen sollte, übersprungen wurde. 


\begin{tabular}{|c|c|c|c|c|c|c|c|c|c|c|}
\hline Vers & Probanden & & & 1 & 2 & 3 & 4 & 5 & 6 & $\mathbf{F}$ \\
\hline & ProbandenNr. & Gesch. & Alter & BAK & BAK & BAK & BAK & BAK & BAK & BAK \\
\hline \multirow{15}{*}{ 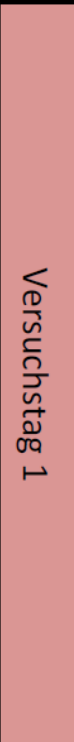 } & 1 & W & 23 & 0,00 & 0,45 & 0,65 & \multirow[t]{2}{*}{1,14} & \multirow[t]{2}{*}{1,71} & & 0,13 \\
\hline & 2 & W & 43 & 0,00 & 0,34 & 0,57 & & & & 0,00 \\
\hline & 3 & W & 33 & 0,00 & 0,25 & 0,57 & \multirow[t]{2}{*}{1,12} & \multirow[t]{3}{*}{1,38} & & 0,00 \\
\hline & 4 & M & 39 & 0,00 & 0,32 & 0,55 & & & & 0,00 \\
\hline & 5 & W & 18 & 0,00 & 0,47 & 1,10 & 1,34 & & & 0,00 \\
\hline & 6 & M & 18 & 0,00 & 0,11 & 0,82 & 1,27 & 1,47 & & 0,03 \\
\hline & 7 & $M$ & 19 & 0,00 & 0,21 & 1,12 & \multirow[t]{2}{*}{1,08} & \multirow[t]{2}{*}{1,50} & & 0,00 \\
\hline & 8 & M & 23 & 0,00 & 0,75 & 1,60 & & & & 0,00 \\
\hline & 9 & W & 38 & 0,00 & 0,27 & 0,52 & 0,96 & 1,47 & & 0,00 \\
\hline & 10 & W & 23 & 0,00 & 0,49 & 0,82 & 1,18 & 1,81 & & 0,20 \\
\hline & 11 & $M$ & 25 & 0,00 & 0,37 & 0,72 & 1,04 & 1,62 & & 0,00 \\
\hline & 12 & M & 30 & 0,00 & 0,49 & 0,83 & 1,37 & 2,58 & & 1,17 \\
\hline & 13 & $M$ & 29 & 0,00 & 0,38 & 0,82 & 1,49 & 2,19 & & 0,40 \\
\hline & 14 & $M$ & 22 & 0,00 & 0,38 & 1,06 & 1,47 & 2,22 & & 0,69 \\
\hline & 15 & $M$ & 22 & 0,02 & 0,44 & 0,70 & 1,29 & 1,73 & & 0,03 \\
\hline \multirow{13}{*}{ 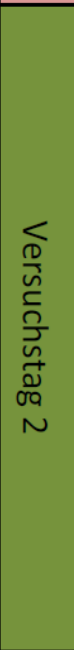 } & 16 & W & 23 & 0,00 & 0,35 & 0,68 & 0,87 & 1,33 & \multirow{4}{*}{1,57} & 0,06 \\
\hline & 17 & $M$ & 22 & 0,00 & 0,35 & 0,73 & 0,95 & 1,27 & & 0,23 \\
\hline & 18 & W & 22 & 0,00 & 0,42 & 0,96 & 1,43 & 1,84 & & 0,79 \\
\hline & 19 & $M$ & 22 & 0,00 & 0,48 & 0,82 & 1,45 & 1,51 & & 0,13 \\
\hline & 20 & W & 23 & 0,00 & 0,42 & 0,72 & 1,00 & 1,26 & \multirow[t]{3}{*}{1,38} & 0,00 \\
\hline & 21 & W & 22 & 0,00 & 0,40 & 0,95 & 0,92 & 1,16 & & 0,00 \\
\hline & 22 & W & 25 & 0,00 & 0,31 & 0,66 & 0,87 & 1,43 & & 0,01 \\
\hline & 23 & $M$ & 34 & 0,00 & 0,38 & 0,93 & 1,40 & 1,80 & \multirow{6}{*}{$\begin{array}{l}2,00 \\
1,49\end{array}$} & 0,21 \\
\hline & 24 & $M$ & 25 & 0,00 & 0,45 & 0,87 & 1,13 & 1,26 & & 0,04 \\
\hline & 25 & W & 22 & 0,00 & 0,44 & 0,66 & 1,15 & 1,66 & & 0,06 \\
\hline & 26 & W & 19 & 0,00 & 0,40 & 0,68 & 1,10 & 1,61 & & 0,26 \\
\hline & 27 & W & 20 & 0,00 & 0,34 & 0,68 & 1,15 & 0,85 & & 0,00 \\
\hline & 28 & $M$ & 23 & 0,00 & 0,21 & 0,30 & & & & 0,00 \\
\hline \multirow{13}{*}{ 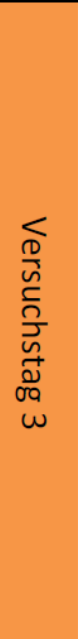 } & 29 & W & 20 & 0,00 & 0,31 & 0,76 & 0,96 & 1,24 & \multirow[t]{4}{*}{1,45} & 0,06 \\
\hline & 30 & $M$ & 53 & 0,00 & 0,38 & 0,71 & 0,98 & 1,27 & & 0,27 \\
\hline & 31 & $M$ & 48 & 0,00 & 0,49 & 0,88 & 1,25 & 1,66 & & 0,74 \\
\hline & 32 & W & 25 & 0,00 & 0,30 & 0,90 & 1,27 & 0,62 & & 0,00 \\
\hline & 33 & W & 22 & 0,00 & 0,32 & 0,92 & 1,35 & 1,71 & 1,33 & 0,00 \\
\hline & 34 & $M$ & 27 & 0,00 & 0,32 & 0,67 & 1,08 & 1,27 & 1,75 & 0,00 \\
\hline & 35 & W & 28 & 0,00 & 0,35 & 0,69 & 1,24 & 1,87 & \multirow[t]{2}{*}{1,57} & 0,27 \\
\hline & 36 & $M$ & 34 & 0,00 & 0,46 & 0,78 & 1,14 & 1,93 & & 0,75 \\
\hline & 37 & $M$ & 24 & 0,00 & 0,36 & 0,72 & 0,91 & 1,22 & \multirow[t]{5}{*}{1,34} & 0,30 \\
\hline & 38 & W & 24 & 0,00 & 0,28 & 0,76 & 1,33 & 1,41 & & 0,00 \\
\hline & 39 & W & 24 & 0,00 & 0,40 & 0,95 & 1,35 & 1,59 & & 0,00 \\
\hline & 40 & $M$ & 28 & 0,00 & 0,29 & 0,66 & 2,06 & \multirow[t]{2}{*}{1,75} & & 0,05 \\
\hline & 41 & W & 41 & 0,00 & 0,45 & 0,70 & 0,80 & & & 0,00 \\
\hline
\end{tabular}

Abbildung 12a:

Blutalkoholkonzentration der Probanden in Bezug auf die Fahrversuche (Spalte „1“: Nüchternfahrversuch; Spalte „F“: Fahrversuch am Folgetag) 


\begin{tabular}{|c|c|c|c|c|c|c|c|c|c|c|}
\hline Vers & Probanden & & & 1 & 2 & 3 & 4 & 5 & 6 & $\mathbf{F}$ \\
\hline & ProbandenNr. & Gesch. & Alter & BAK & BAK & BAK & BAK & BAK & BAK & BAK \\
\hline \multirow{18}{*}{ 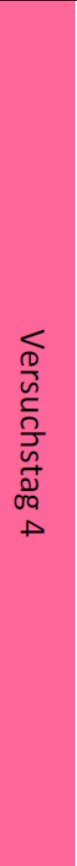 } & 42 & $M$ & 30 & 0,00 & 0,38 & 1,10 & 1,50 & & & 0,05 \\
\hline & 43 & W & 28 & 0,00 & 0,32 & 0,78 & 1,56 & 1,55 & & 0,00 \\
\hline & 44 & W & 25 & 0,00 & 0,22 & 0,83 & 1,06 & 1,33 & & 0,00 \\
\hline & 45 & W & 22 & 0,00 & 0,22 & 0,66 & 0,88 & 1,14 & 1,04 & 0,00 \\
\hline & 46 & W & 23 & 0,00 & 0,27 & 0,68 & 0,86 & 1,09 & 1,05 & 0,00 \\
\hline & 47 & W & 22 & 0,00 & 0,23 & 0,72 & 0,92 & 1,34 & 1,75 & 0,15 \\
\hline & 48 & W & 22 & 0,00 & 0,29 & 0,69 & 0,98 & 1,28 & 1,82 & 0,18 \\
\hline & 49 & W & 24 & 0,00 & 0,18 & 0,88 & 1,20 & 1,61 & 1,95 & 0,46 \\
\hline & 50 & $M$ & 25 & 0,00 & 0,37 & 0,65 & 1,12 & 0,87 & & 0,00 \\
\hline & 51 & $M$ & 25 & 0,00 & 0,09 & 0,56 & 1,44 & & & 0,00 \\
\hline & 52 & W & 23 & 0,00 & 0,19 & 0,57 & 0,84 & 0,93 & & 0,00 \\
\hline & 53 & $M$ & 26 & 0,00 & 0,07 & 0,51 & 1,23 & 1,49 & & 0,00 \\
\hline & 54 & $M$ & 31 & 0,00 & 0,17 & 0,89 & 1,28 & 1,51 & & 0,11 \\
\hline & 55 & $M$ & 24 & 0,00 & 0,07 & 0,60 & 0,99 & 1,20 & & 0,00 \\
\hline & 56 & $M$ & 44 & 0,00 & 0,16 & 0,58 & 1,09 & 1,41 & 1,92 & 0,40 \\
\hline & 57 & W & 30 & 0,00 & 0,34 & 0,82 & & & & 0,00 \\
\hline & 58 & W & 30 & 0,00 & 0,10 & 0,68 & 0,86 & 0,79 & & 0,00 \\
\hline & 59 & W & 33 & 0,00 & 0,12 & 0,46 & & & & 0,00 \\
\hline \multirow{15}{*}{ 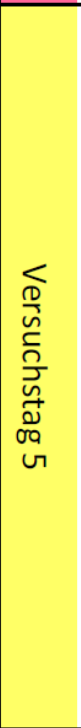 } & 60 & $M$ & 26 & 0,00 & 0,72 & 1,32 & 1,97 & 1,91 & \multirow{15}{*}{$\begin{array}{l}1,96 \\
1,36\end{array}$} & 0,50 \\
\hline & 61 & $M$ & 21 & 0,00 & 0,52 & 1,10 & 1,37 & 1,39 & & 0,00 \\
\hline & 62 & W & 26 & 0,00 & 0,35 & 0,92 & 1,56 & 1,40 & & 0,00 \\
\hline & 63 & W & 25 & 0,00 & 0,29 & 1,17 & 1,34 & & & 0,00 \\
\hline & 64 & w & 25 & 0,00 & 0,27 & 0,88 & 1,24 & & & 0,00 \\
\hline & 65 & $M$ & 27 & 0,00 & 0,25 & 0,71 & 0,94 & 0,96 & & 0,00 \\
\hline & 66 & $M$ & 26 & 0,00 & 0,32 & 0,59 & 0,97 & 1,35 & & 0,12 \\
\hline & 67 & w & 20 & 0,00 & 0,70 & 1,55 & 1,86 & 2,05 & & 0,11 \\
\hline & 68 & $M$ & 26 & 0,00 & 0,49 & 0,92 & 1,51 & 1,91 & & 0,16 \\
\hline & 69 & $M$ & 21 & 0,00 & 0,88 & 1,26 & 1,90 & 2,01 & & 0,44 \\
\hline & 70 & $M$ & 50 & 0,00 & 0,41 & 0,61 & 0,97 & 1,43 & & 0,51 \\
\hline & 71 & $M$ & 30 & 0,00 & 0,28 & 0,97 & 1,57 & 2,30 & & 0,77 \\
\hline & 72 & $M$ & 32 & 0,00 & 0,16 & 0,50 & 0,84 & 1,50 & & 0,00 \\
\hline & 73 & $M$ & 35 & 0,00 & 0,43 & 0,75 & 2,27 & & & \\
\hline & 74 & $M$ & 35 & 0,00 & 0,48 & 1,26 & 1,53 & & & \\
\hline \multirow{9}{*}{ 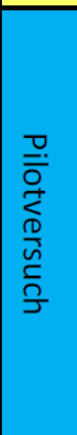 } & 101 & W & 25 & 0,00 & 0,29 & 1,02 & 1,26 & 1,45 & \multirow{7}{*}{1,93} & \\
\hline & 102 & $M$ & 34 & 0,00 & 0,32 & 0,58 & 1,18 & 2,18 & & \\
\hline & 103 & $M$ & 24 & 0,00 & 0,30 & 0,93 & 1,18 & 1,56 & & \\
\hline & 104 & $M$ & 25 & 0,00 & 0,30 & 0,74 & \multirow{4}{*}{0,00} & & & \\
\hline & 105 & $M$ & 20 & 0,00 & 0,21 & 0,38 & & & & \\
\hline & 106 & $M$ & 39 & 0,00 & 0,07 & 0,01 & & & & \\
\hline & 107 & W & 18 & 0,00 & 0,68 & 1,04 & & & & \\
\hline & 108 & w & 26 & 0,00 & 0,36 & 0,54 & 1,01 & 1,29 & \multirow[t]{2}{*}{1,48} & \\
\hline & 109 & $\mathrm{M}$ & 19 & 0,00 & 0,64 & 1,16 & 1,40 & 1,56 & & \\
\hline
\end{tabular}

Abbildung 12b:

Blutalkoholkonzentration der Probanden in Bezug auf die Fahrversuche (Spalte „1“: Nüchternfahrversuch; Spalte „F“: Fahrversuch am Folgetag) 
Andererseits kam es auch vor, dass ein BAK-Bereich über einen längeren Zeitraum nicht überschritten werden konnte, so dass ein Proband innerhalb eines Zielbereiches zwei Fahrversuche absolvierte. So lag beispielsweise im Rahmen der zweiten Testreihe (erster Versuch in alkoholisiertem Zustand) die niedrigste BAK bei $0,07 \%$, die höchste hingegen bei 0,88 \%o.

Für die weitere Beurteilung der Fahrleistung wurde daher lediglich die ermittelte BAK zu Grunde gelegt, unabhängig von der Anzahl der bis zu diesem Zeitpunkt vorgenommenen Fahrversuche. Dies bedeutet, dass ein Proband innerhalb eines BAK-Korridors auch mehrere bzw. keinen Fahrversuch vorgenommen haben konnte.

Zu den Details der ermittelten BAK im Rahmen der Fahrversuche sei auf die Abbildungen 12a und $12 \mathrm{~b}$ verwiesen. Bei Proband 2 konnten aufgrund erheblicher Probleme bei den venösen Blutentnahmen nach dem dritten Fahrversuch keine weiteren Blutentnahmen vorgenommen werden, so dass bis zum Folgemorgen keine weiteren Testreihen erfolgten.

\subsection{Ergebnisse der ärztlichen Untersuchungen im Rahmen der Blutentnahmen}

Einerseits wurden die bei der ärztlichen Untersuchung festgestellten Ausfallerscheinungen ausgewertet; andererseits wurden auch von den Probanden subjektiv geäußerte Beeinträchtigungen des Wohlbefindens oder der Leistungsfähigkeit notiert. Diese von den Probanden subjektiv geäußerten, stark variablen Symptome wurden zusätzlich, jedoch separat ausgewertet, um Hinweise auf eine mögliche subjektive, alkoholbedingte Beeinträchtigung der Fahrsicherheit nicht zu versäumen.

Alle Befunde wurden hinsichtlich ihrer verkehrsmedizinischen Relevanz mittels Punktvergabe unterschiedlich gewichtet. Zur Vermeidung einer zu hohen Punktvergabe bei unterschiedlichen Symptomen, die jedoch auf dieselbe Ursache rückschließen ließen, wurde die Punktvergabe teilweise "gedeckelt“. So wurden die unter Bewusstsein, Sprechen/ formaler Denkablauf, subjektivem Befinden und Stimmung zusammengefassten Befunde auf jeweils maximal 3 Punkte "gedeckelt“. Ferner wurden die auf Kreislaufstörungen hinweisenden Befunde (Zittern, stattgehabtes Erbrechen, Hautblässe, Hauttrockenheit, diffuses Unwohlsein) auf maximal 2 Punkte „gedeckelt". Folgende weitere Punktwertvergaben erfolgten: 
Hinsichtlich der Finger-Finger-Probe, der Nasen-Finger-Probe und der plötzlichen Kehrtwendung (nach vorherigem Gehen) wurde folgende Punktvergabe vorgenommen:

- sicher: 0

- grenzwertig: 0,5

- unsicher: 1

Hinsichtlich des Gangs (geradeaus) wurde folgende Punktvergabe vorgenommen:

- sicher: 0

- schleppend: 1

- schwankend / torkelnd: 2

Hinsichtlich des Bewusstseins wurde folgende Punktvergabe vorgenommen:

- klar: 0

- benommen / somnolent: 1

- verwirrt: 2

- bewusstlos: 4

Hinsichtlich der Sprache wurde folgende Punktvergabe vorgenommen:

- deutlich: 0

- grenzwertig: 0,5

- „schwere Zunge“: 1

- verwaschen / lallend: 2

Hinsichtlich des Sprechens / formalen Denkablaufs wurde folgende Punktvergabe vorgenommen:

- geordnet: 0

- $\quad$ verlangsamt / beschleunigt / haftend / perseverierend / sprunghaft bzw. ideenflüchtig: 1

- verworren / zerfahren: 2 
Hinsichtlich des subjektiven Befindens wurde folgende Punktvergabe vorgenommen:

- normal: 0

- Schweißausbruch bzw. Hitzegefühl / Kältegefühl / Kopfschmerzen / Mundtrockenheit / Durst / Müdigkeit bzw. Gähnen: 1

- Übelkeit / Schwindel / schwerfälliges oder schläfriges Verhalten: 2

Hinsichtlich der Stimmung wurde folgende Punktvergabe vorgenommen:

- ruhig: 0

- distanzlos / fröhlich / redselig bzw. logorrhoeisch / aufgeregt bzw. nervös / abweisend bzw. distanziert: 1

- $\quad$ provokativ bzw. enthemmt / aggressiv bzw. übergriffig: 2

Hinsichtlich der Pupillen wurde folgende Punktvergabe vorgenommen:

- Unauffällig: 0

- lichtstarr / lichtstarr erweitert / lichtstarr verengt: 1

Hinsichtlich der Bindehäute wurde folgende Punktvergabe vorgenommen:

- klar: 0

- gerötet / geschwollen: 1

Hinsichtlich des äußerlichen Eindrucks des Alkoholisierungsgrades wurde folgende Punktvergabe vorgenommen:

- nicht merkbar: 0

- leicht: 1

- leicht bis deutlich: 1,5

- deutlich: 2

- deutlich bis stark: 2,5

- $\quad$ stark: 3 
Hinsichtlich der zusätzlichen ärztlichen Notizen (freies Textfeld) wurden die folgenden Befunde erfasst und wie folgt gewichtet:

- lässt Sachen fallen / allgemein verlangsamtes Verhalten / Unschärfe beim Sehen bzw. Doppelbilder / Dysmetrie (Bewegungsstörung) / starke Selbstüberschätzung / gesteigertes Selbstbewusstsein / Filmriss bzw. Erinnerungslücke: 2

- Taubheitsgefühl Finger, Hände und bzw. oder Zunge / Konzentrationsschwäche / glasiger Blick / redet bzw. lacht laut und bzw. oder viel / Kreislaufprobleme / Zittern / Erbrechen / läppischer Affekt / trockene Haut / Tonuserhöhung im Gesicht / blasses Hautkolorit / Magenprobleme bzw. -schmerzen / stumpfes bzw. lethargisches Verhalten / weinerliches Verhalten bzw. Weinen / Euphorie / Vortanzen / Affektlabilität / Affektinkontinenz bzw. aufbrausendes Verhalten / starke Dysarthrie (Sprechstörung) / feuchte Aussprache / psychisch überlagertes Verhalten / Histrionie (theatralisches Verhalten) / Reflux (Sodbrennen) / „Schluck-auf“ / Störung des Affekts / diffuses Unwohlsein: 1

Die Auswertung des „Grades der Alkoholisierung“, wie er sich aus dem subjektiven Eindruck des blutabnehmenden Arztes ergab, zeigte eine exponentielle Zunahme mit steigender BAK (s. Abb. 13). Dies spricht dafür, dass niedrigere und mittlere BAK von den Probanden weitaus besser kompensiert werden konnten als die höheren BAK. Bereits in dem BAK-Bereich zwischen 0,21 und $0,40 \%$ zeigte sich eine signifikante Veränderung dieses ärztlichen Eindrucks im Vergleich zu der nüchternen Probandenuntersuchung $(p=0,00031)$. Allerdings war dem jeweiligen Arzt die AAK bei der Untersuchung bekannt, und der Untersucher hatte zusätzlich die individuelle Veränderung des Zustands der Probanden im Verlauf des Versuchs registriert. 


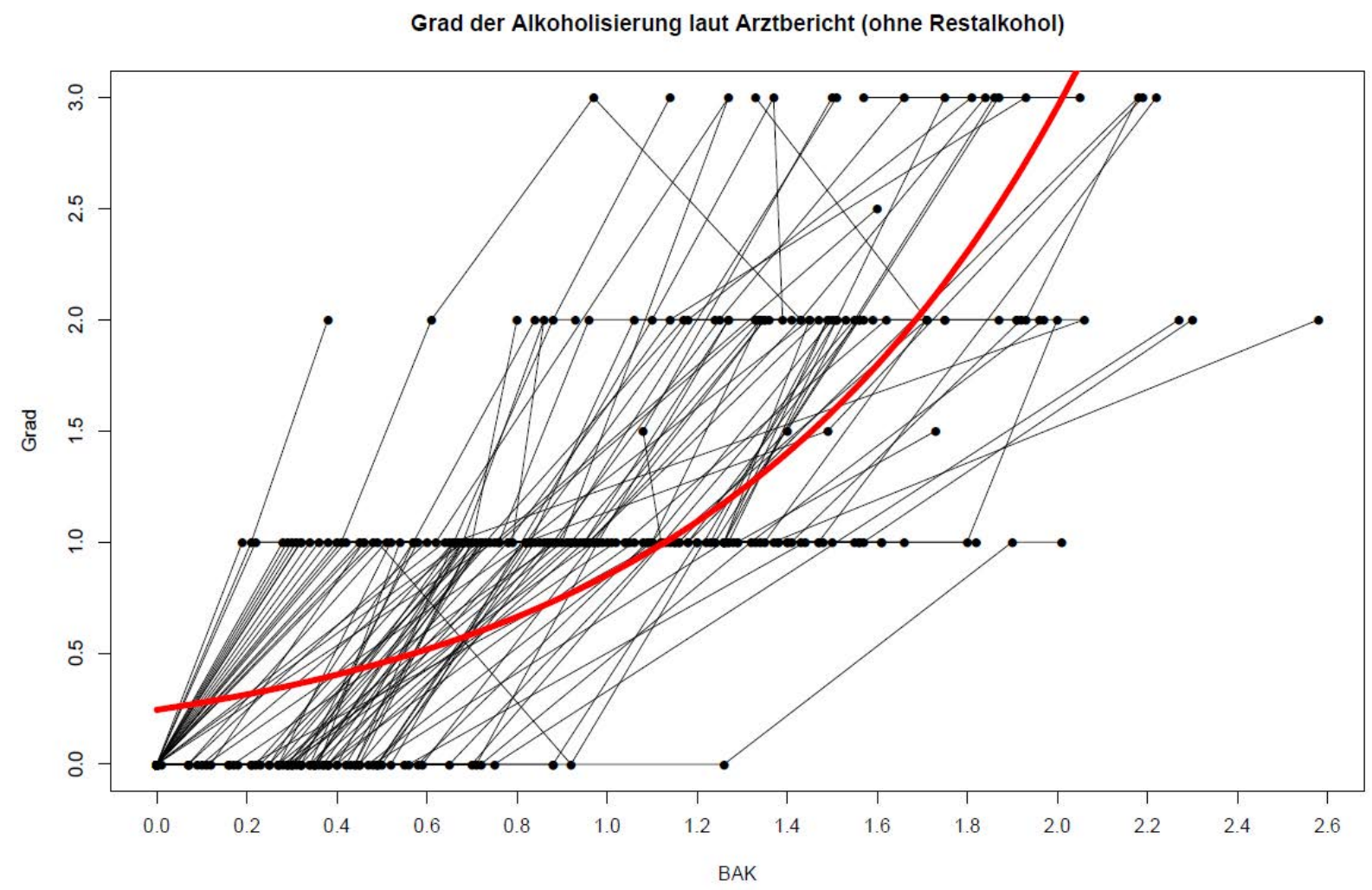

Abbildung 13:

Grad der Alkoholisierung (ohne Restalkohol) in Bezug auf die BAK

Bei der Auswertung des ärztlichen Berichts in Bezug auf motorische Defizite (Finger-Finger-Probe, Nasen-Finger-Probe, Gang, plötzliche Kehrtwendung) und Zeichen einer psychischen Beeinträchtigung (Bewusstsein, Denkablauf, Sprache, Befinden, Stimmung) nach obiger Punktvergabe (sog. Bericht 1) zeigte sich, dass oberhalb einer BAK von 1,0\%o lediglich ein Proband keinerlei Ausfälle aufwies (Abb. 14). Die Abb. 14 beinhaltet ausschließlich Auffälligkeiten, die in jeder ärztlichen Untersuchung (mit kooperativen Probanden) erhoben werden und gleichzeitig unmittelbaren Einfluss auf die Fahreigenschaften haben können. Insgesamt liegt auch hier eine exponentielle Zunahme dieser ärztlich dokumentierten Ausfälle mit steigender BAK vor, was für vorhandene Kompensationsmöglichkeiten dieser Ausfälle bei niedrigeren BAK spricht. Im Vergleich zum Nüchternzustand zeigten bereits die nur gering alkoholbelasteten Probanden (bis $0,20 \%$ ) eine signifikante Zunahme von Ausfällen ( $p=0,047)$ : 


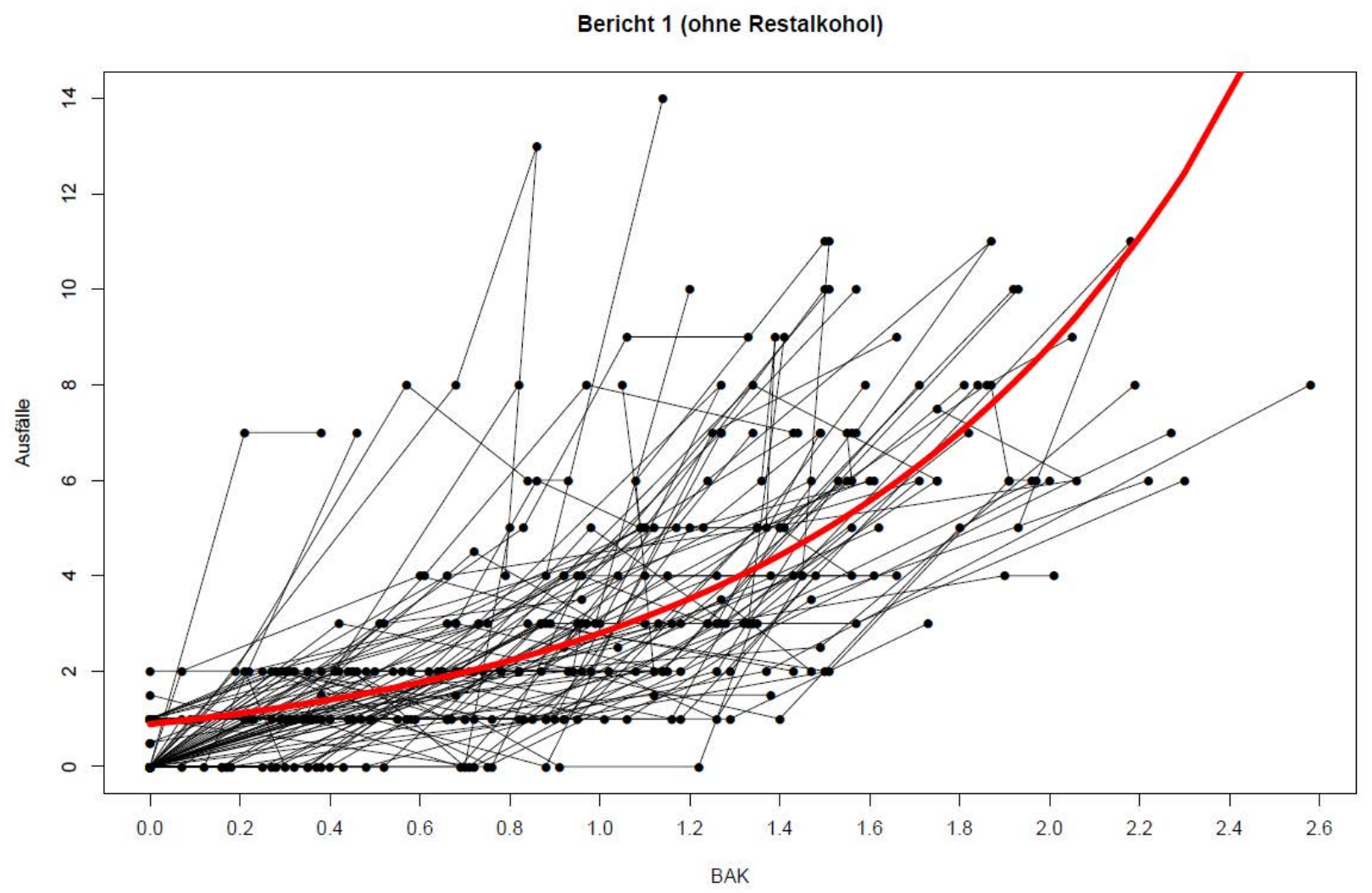

Abbildung 14:

Anzahl ärztlich dokumentierter Ausfälle in Bezug auf die BAK (ohne Restalkohol), Bericht 1

Ein weniger eindeutiges Bild zeichnete sich bei der Betrachtung der übrigen im ärztlichen Bericht im Rahmen einer Blutentnahme zu erhebenden Befunde ab (sog. Bericht 2: freies Textfeld, Bindehäute, Pupillen-Lichtreaktion). Um Redundanzen zu vermeiden, wurden Befunde, die auf eine Kreislaufstörung hinweisen - wie oben ausgeführt - „gedeckelt“, wobei es unerheblich war, ob diese im Standardbericht oder im freien Textfeld erfasst wurden. Es zeigte sich, dass mehrere Probanden auch weit jenseits einer BAK von 1,6\% keine solchen Auffälligkeiten aufwiesen (Abb. 15). Dennoch zeigten bereits Probanden mit einer BAK zwischen 0,21 und $0,40 \%$ signifikant mehr Auffälligkeiten als nüchterne Probanden $(p=0,026)$.

Bei der Kombination dieser drei im ärztlichen Bericht dokumentierten Auffälligkeiten zeigt sich bis zu einem Bereich von etwa 1,0\% eine annähernd lineare Zunahme der dokumentierten Auffälligkeiten, die bei höheren BAK exponentiellen Charakter bekommt (Abb. 16). 


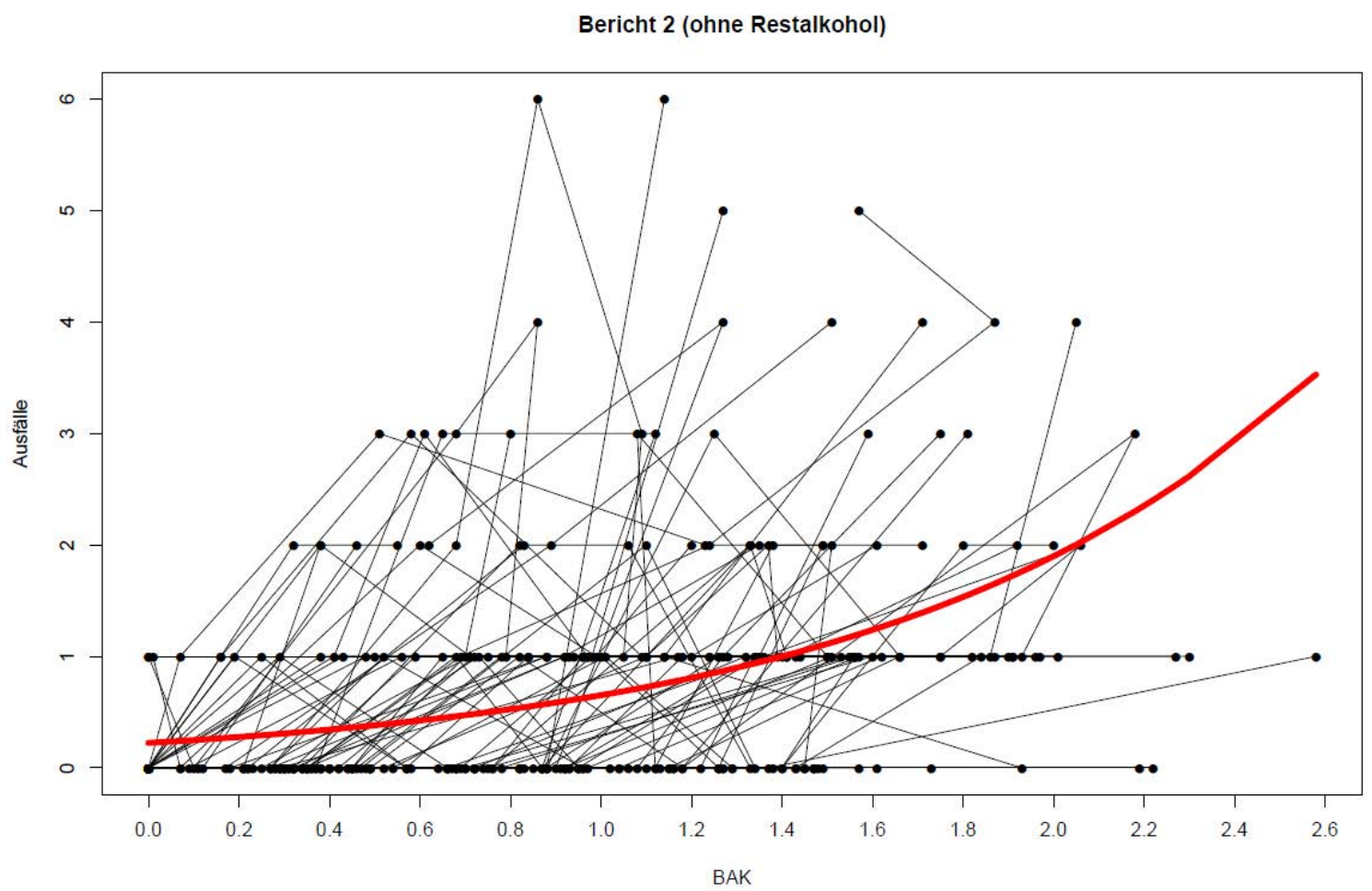

Abbildung 15:

Anzahl ärztlich dokumentierter Ausfälle in Bezug auf die BAK (ohne Restalkohol), Bericht 2

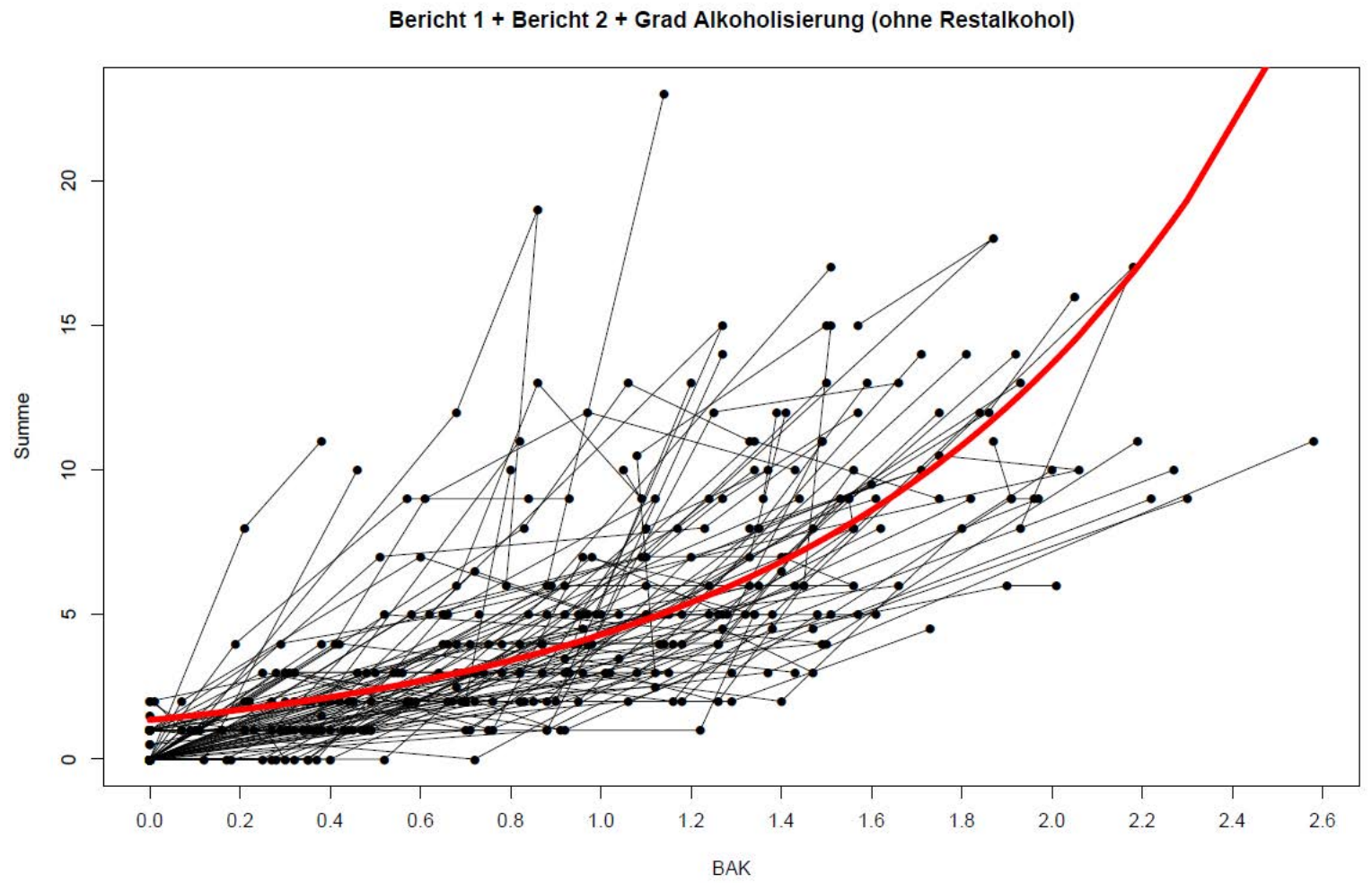

Abbildung 16:

Anzahl aller ärztlich dokumentierten Ausfälle in Bezug auf die BAK (ohne Restalkohol), Berichte 1 und 2 sowie Grad der Alkoholisierung laut Arztbericht 


\subsection{Ergebnisse der augenärztlichen Untersuchungen}

In dem Swinging-Test, bei dem der Proband den sich abwechselnd seitwärts bewegenden Zeigefinger des untersuchenden Augenarztes zehn Mal berühren musste, zeigte sich kein statistisch signifikanter Zusammenhang zwischen der benötigten Zeit und der BAK (Abb. 17):

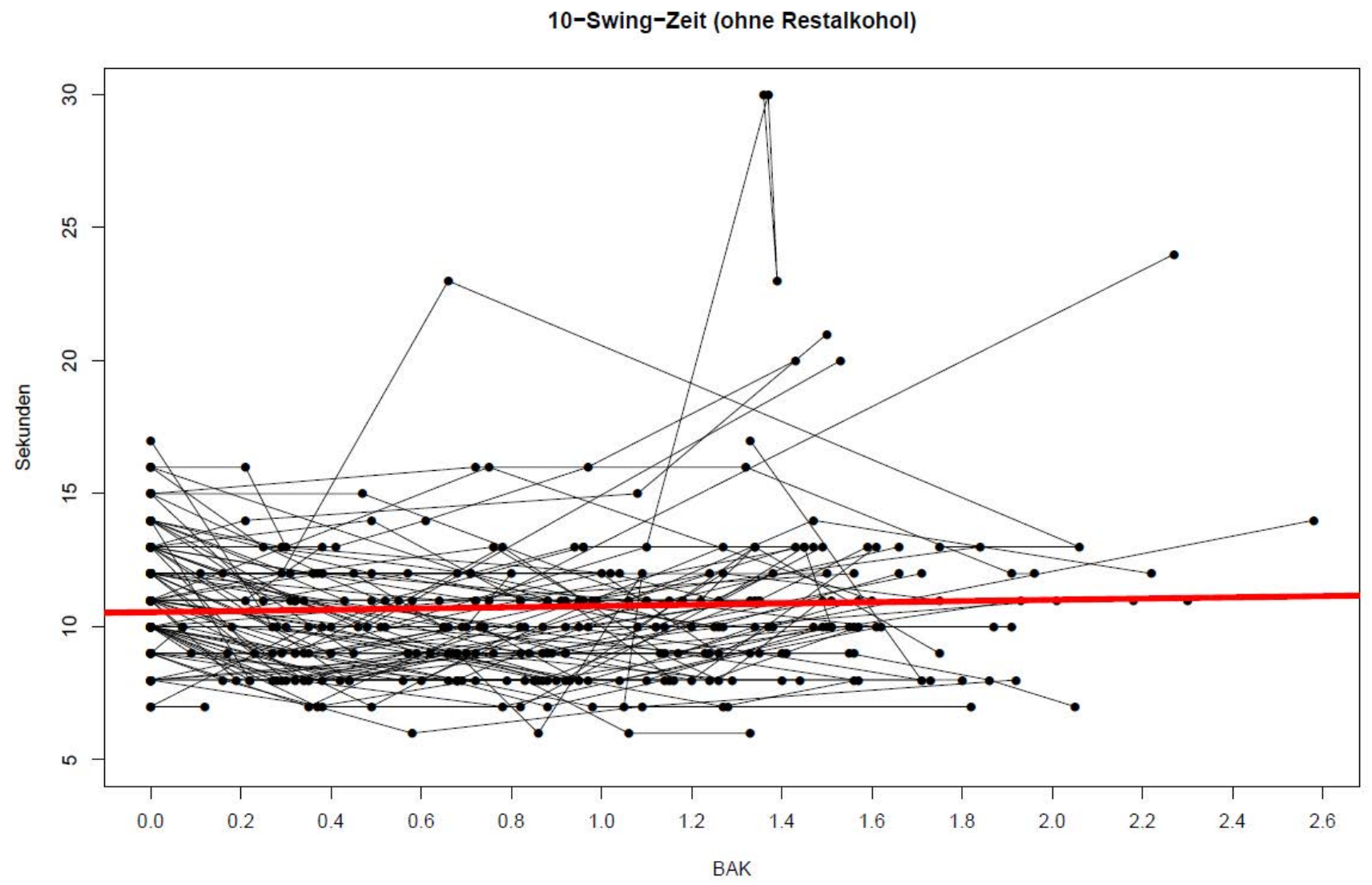

\section{Abbildung 17:}

Benötigte Zeit für den Swinging-Test in Bezug auf die BAK (ohne Restalkohol)

Hingegen nahm die Zeit, die zum Vorlesen des definierten Textabschnittes benötigt wurde, mit steigender BAK zu, wobei eine statistisch signifikante Veränderung erst bei BAK von mehr als $1,0 \%$ zu erkennen war ( $p=0,00056 ;$ Abb. 18): 


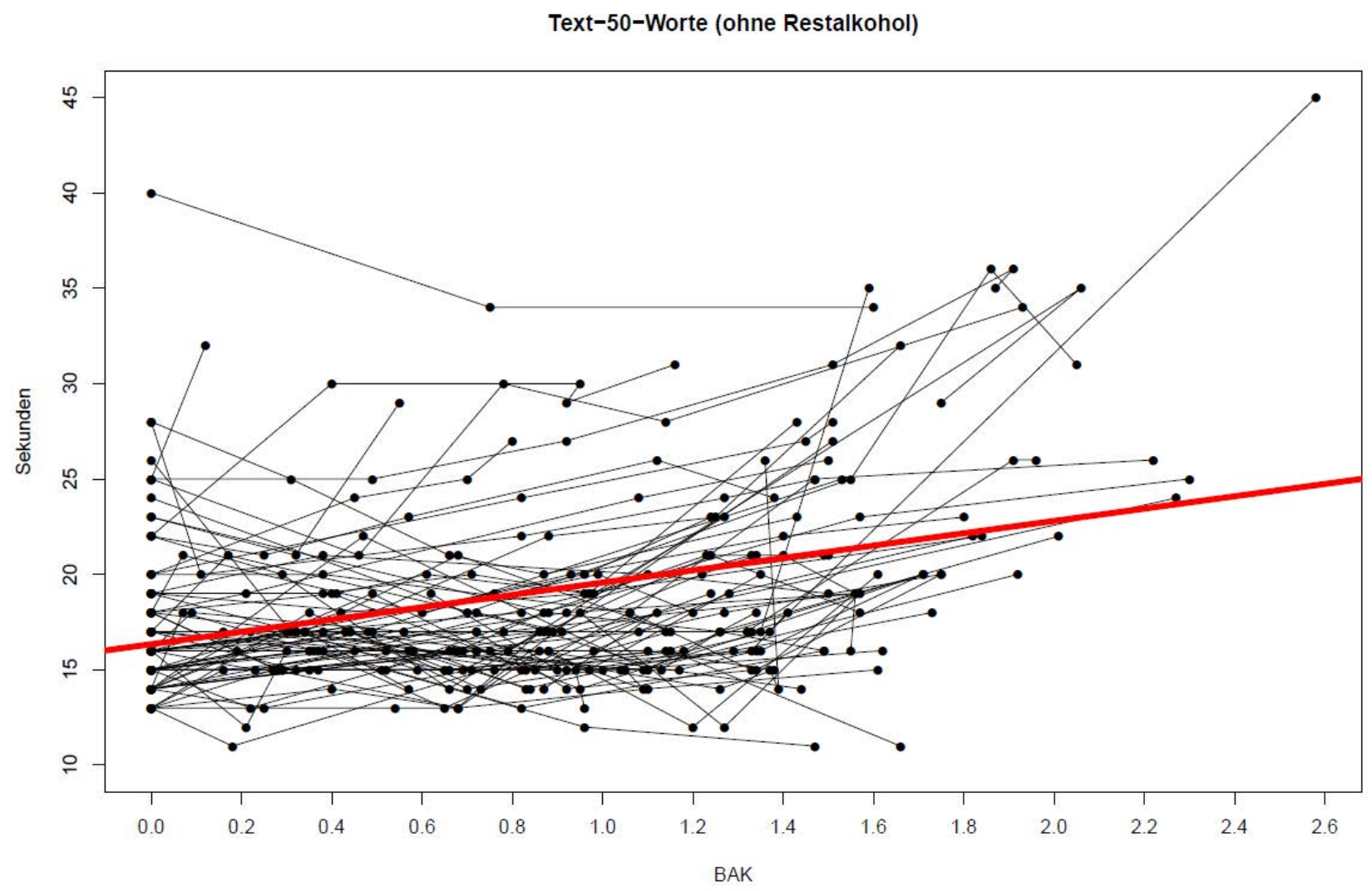

Abbildung 18:

Benötigte Zeit zum Vorlesen eines definierten Textabschnittes in Bezug auf die BAK

Es zeigte sich ferner eine lineare Abnahme der gesamten Fusionsbreite (Abb. 19), wobei die Probanden ab einer BAK von 1,2\% eine signifikant niedrigere Fusionsbreite als im Nüchternversuch aufwiesen ( $p=0,0020)$. Die Resultate zeigten dabei eine erhebliche Streubreite. 


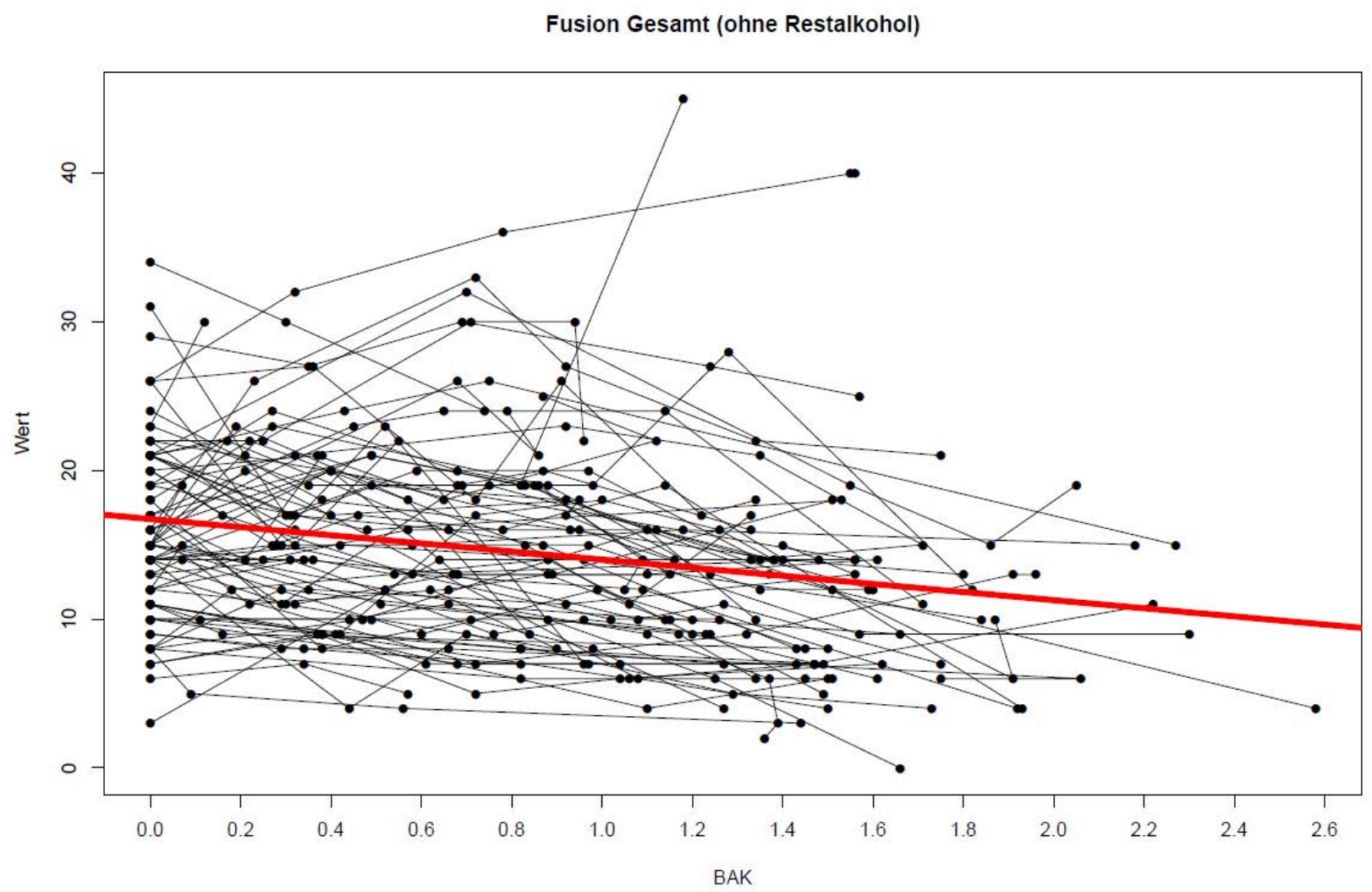

Abbildung 19:

Veränderung der gesamten Fusionsbreite (Wert in Dioptrie) in Bezug auf die BAK

\subsection{Ergebnisse der Videoanalysen der Fahrversuche}

Durch die Videoaufzeichnungen konnten u.a. während der Fahrt begangene Linienberührungen, das Reaktionsvermögen auf verschiedene Ereignisse sowie der Gleichgewichtssinn beurteilt werden.

Zunächst sei darauf verwiesen, dass die Fahrversuche folgender Probanden unterbrochen bzw. abgebrochen werden mussten:

- Bei Proband 8 musste der 3. Fahrversuch beim Durchfahren des Slalomparcours wegen Fahrunfähigkeit durch den Versuchsleiter abgebrochen werden (BAK: 1,6\%o). Dieser Proband stand jedoch, wie die nachträgliche Untersuchung seiner Blutprobe ergab, zusätzlich unter Cannabiseinfluss; es kann aufgrund der Befunde unterstellt werden, dass es während der Versuche zu einem heimlichen Cannabisgebrauch durch diesen Probanden kam. 
- Bei Proband 10 musste der 5. Fahrversuch aufgrund von Anfahrschwierigkeiten nach Anhalten an der Lichtzeichenanlage durch den Versuchsleiter abgebrochen werden (BAK: $1,81 \%$ ).

- Die Probanden 29 und 45 brachen jeweils nach dem 5. Fahrversuch aufgrund von subjektivem Unwohlsein den Versuch ab (BAK: 1,45 \%o bzw. 1,04 \%).

- Proband 49 durfte am 6. Fahrversuch aufgrund starker alkoholbedingter Ausfallerscheinungen nicht teilnehmen (BAK: 1,95\%).

- Proband 57 brach nach dem 3. Fahrversuch auf eigenen Wunsch aufgrund von subjektivem Unwohlsein ab (BAK 0,82 \%o).

- Proband 59 brach nach dem 3. Fahrversuch auf eigenen Wunsch aufgrund von subjektivem Unwohlsein ab (BAK 0,46\%o).

- Die Probanden 73 (BAK 2,23\%o) und 74 (BAK 1,53\%) wurden wegen aggressiver und unkooperativer Verhaltensweisen, die nicht mit der Versuchsdurchführung vereinbar waren, nach dem 4. Fahrversuch um 22:30 Uhr vom weiteren Versuchsablauf ausgeschlossen und zu ihrer Wohnanschrift begleitet.

Die Videoauswertung der Fahrversuche erfolgte nach den folgenden drei Kriterien:

- Koordinationsfehler,

- Konzentrationsfehler,

- Reaktionsfehler.

Die einzelnen Fehler wurden dabei hinsichtlich ihrer verkehrsmedizinischen Relevanz mittels der Vergabe von Fehlerpunkten unterschiedlich gewichtet.

Unter Koordinationsfehlern wurden Linienberührungen und -überfahrungen, das Berühren, Auslassen oder Umwerfen von Hindernissen, das Absetzen eines oder beider Füße an nicht vorgesehener Stelle, Anfahrtsschwierigkeiten sowie das Fahren von Schlangenlinien verstanden. Folgende Punktwerte wurden für die jeweiligen Koordinationsfehler vergeben:

- Linienüberfahrung auf gerader Strecke: 2 Fehlerpunkte.

- Spur verlassen auf gerader Strecke: 3 Fehlerpunkte.

- Linienüberfahrung im Kreisverkehr: 2 Fehlerpunkte.

- Spur verlassen im Kreisverkehr: 3 Fehlerpunkte.

- Berührung einer Slalomstange / Pylone: 1 Fehlerpunkt.

- Umwerfen einer Slalomstange / Pylone: 2 Fehlerpunkte. 
- Berührung eines Slalomhütchens: 1 Fehlerpunkt.

- Auslassen eines Hindernisses: 2 Fehlerpunkte.

- Absetzen eines Fußes / beider Füße: 1 Fehlerpunkt.

- Anfahrtsschwierigkeiten: 2 Fehlerpunkte.

- Leichte Schlangenlinien: 1 Fehlerpunkt.

- Deutliche Schlangenlinien: 2 Fehlerpunkte.

- Umwerfen bzw. Überfahren der Slalomhütchen: 2 Fehlerpunkte.

- Berührung einer blauen Tonne (Durchfahren der Gasse): 1 Fehlerpunkt.

- Umwerfen einer blauen Tonne (Durchfahren der Gasse): 3 Fehlerpunkte.

Unter Konzentrationsfehlern wurden inadäquates Verhalten an der Lichtzeichenanlage und den vorgesehenen Stopplinien sowie die zumindest teilweise fehlerhafte Wiedergabe des während des Fahrversuchs zu merkenden Wortes angesehen. Folgende Punktwerte wurden für die jeweiligen Konzentrationsfehler vergeben:

- Fahren bei gelber Ampel: 1 Fehlerpunkt.

- Fahren bei roter Ampel: 3 Fehlerpunkte.

- Warten bei grüner Ampel ohne ersichtlichen Grund: 1 Fehlerpunkt.

- Überfahren einer Stopplinie: 2 Fehlerpunkte.

- Für jede von der vorgegebenen Kreisfahrtenanzahl (=3) abweichende Runde: 1 Fehlerpunkt.

- Wort vergessen / falsch oder nicht abgelesen: 1 Fehlerpunkt.

- Wort teilweise richtig: 0,5 Fehlerpunkte.

Unter einem Reaktionsfehler wurde die spontane, inadäquate Reaktion auf das ggf. während der Fahrt auftretende, „überraschende Ereignis“ (s. Punkt 2.3.) verstanden. Hierbei wurde lediglich zwischen adäquater und inadäquater Reaktion unterschieden. Ein Reaktionsfehler wurde mit 3 Fehlerpunkten belegt. Bei den 83 Probanden wurden insgesamt acht inadäquate Reaktionen registriert:

- Proband Nr. 2 / Fahrversuch 2 / 0,34 \%: Der Proband versuchte während der ungebremsten Fahrt den vor das Fahrrad rollenden Ball mit dem Fuß wegzuschießen.

- Proband Nr. 10 / Fahrversuch 4/1,18 \%o: Der Proband fuhr gegen den vor das Fahrrad rollenden Ball. 
- Proband Nr. 11 / Fahrversuch 4/1,04 \%o: Der Proband fuhr ungebremst auf die Personen zu, die sich auf der Fahrbahn befanden, so dass diese zur Seite springen mussten.

- Proband Nr. 22 / Fahrversuch 2 / 0,31 \%o: Der Proband reagierte unsicher und zögerlich auf die auf der Fahrbahn stehenden Personen.

- Proband Nr. 47 / Fahrversuch $3 /$ 0,72 \%o: Der Proband konnte nicht adäquat auf die kurzfristig verschobene Tonne reagieren (unsichere Weiterfahrt trotz Gegenstand auf der Fahrbahn).

- Proband Nr. 54 / Fahrversuch am Folgemorgen / 0,00 \%o: Der Proband trat mit dem Fuß gegen die kurzfristig verschobene Tonne, statt ihr auszuweichen.

- Proband Nr. 61 / Fahrversuch 6 / 1,36 \%: Der Proband fuhr gegen eine kurzfristig verschobene Tonne.

- Proband Nr. 73 / Fahrversuch 4 / 2,27 \%o: Vollständig unkontrollierte Fahrt ohne Beachtung von Verkehrszeichen oder Fahrspuren.

Zunächst sollen an dieser Stelle die Fehler betrachtet werden, die als "grobe Fahrfehler“ bezeichnet wurden (Abb. 20a und b). Hiermit sind Koordinationsfehler gemeint, bei denen eine unmittelbare Gefährdung des Straßenverkehrs wahrscheinlich erschien (Fahren von Schlangenlinien, vollständiges Verlassen der Fahrspur, Anfahrschwierigkeiten). Alle anderen Koordinationsfehler wurden als „allgemeine Fahrfehler“ klassifiziert. Ab einer BAK von mehr als $0,8 \%$ zeigte sich eine hochsignifikante Zunahme dieser „groben Fahrfehler“ im Vergleich zur Nüchternfahrt $(p=0,0042)$. Bemerkenswert ist auch hier, dass vereinzelte Probanden selbst bei sehr hohen BAK (z.B. mehr als 1,6 \%o) keine groben Fahrfehler machten. 


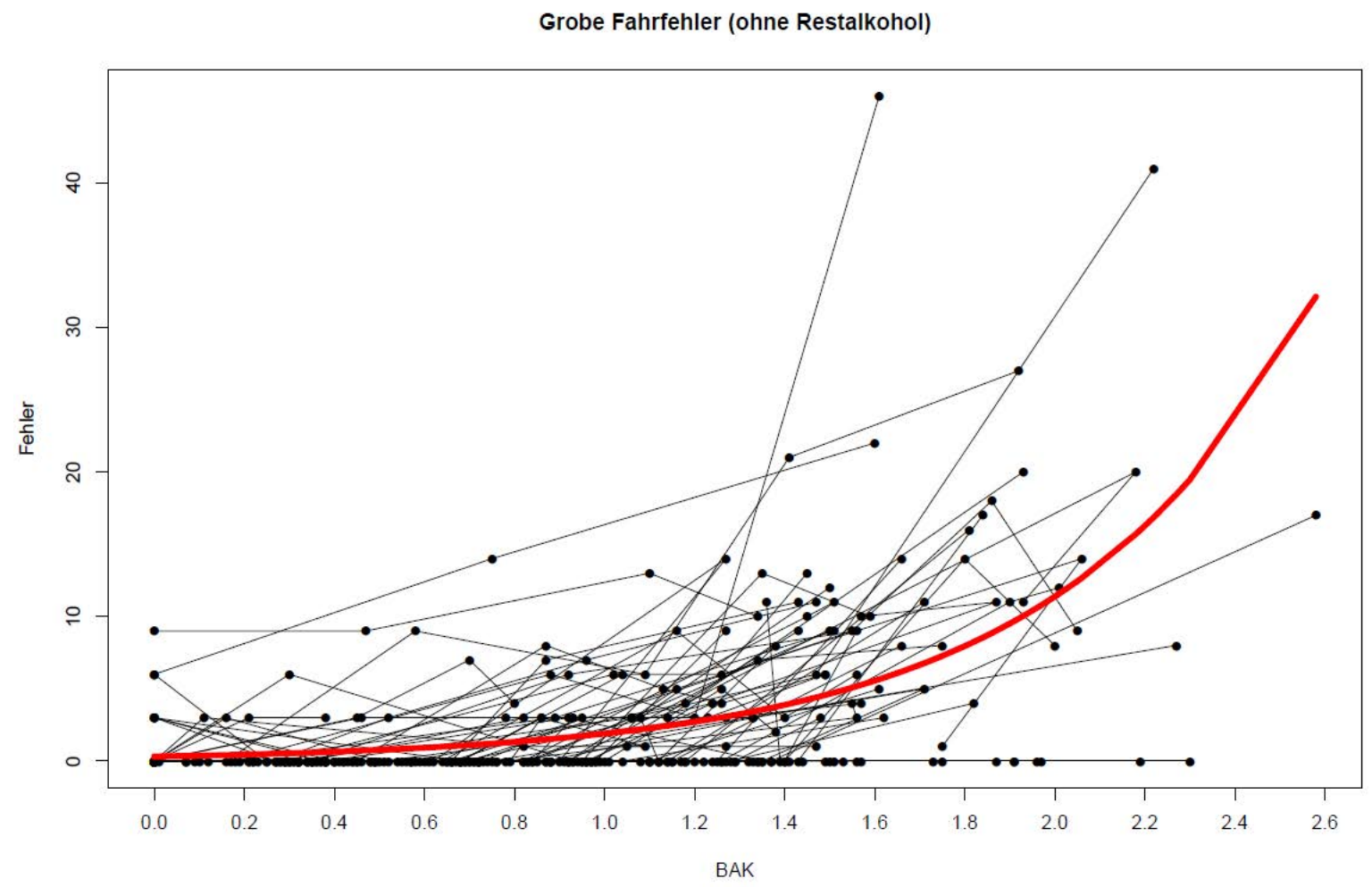

Abbildung 20a:

„Grobe“ Fahrfehler (Koordinationsfehler) in Bezug auf die BAK

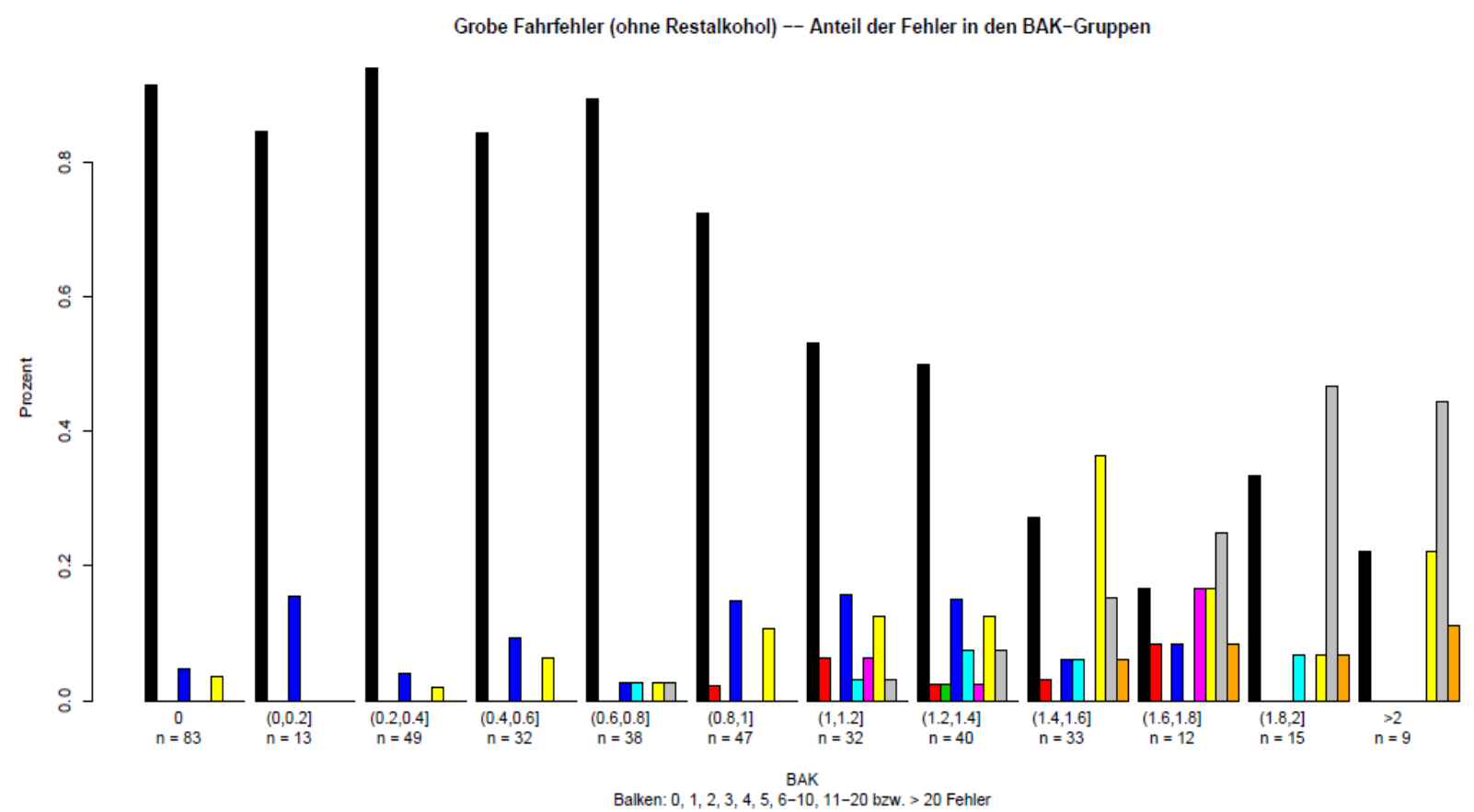

Abbildung $20 \mathrm{~b}$ :

Anteil von Probanden mit „groben“ Fahrfehlern (Koordinationsfehler) in Bezug auf die BAK (Balkenfarbe in Fehlerpunkten: schwarz 0, rot 1, grün 2, blau 3, türkis 4, rosa 5, gelb 6-10, grau $11-20$, orange $>20$ ) 
Wenn man hingegen alle oben aufgeführten Koordinationsfehler betrachtet („Allgemeine und grobe Fahrfehler"; Abb. 21a und b), zeigt sich eine signifikante Fehlerzunahme bereits bei einer geringen Alkoholisierung bis zu einer BAK von 0,20\%o $(p=0,02)$.

Beim Kollektivvergleich der Fahrten unter starkem Alkoholeinfluss (siehe Abb. 21b) mit den Nüchternfahrten zeigt sich, dass es einige wenige Probanden gibt, die auch unter erheblichem Alkoholeinfluss weniger Fahrfehler begehen als andere Probanden im Nüchternzustand. Die große Mehrzahl der Probanden zeigte aber schon bei deutlich niedrigeren BAK-Werten eine Zunahme der Fahrfehler gegenüber der Leistung im nüchternen Zustand. So lagen im BAK-Korridor zwischen 0,8 und 1,0 \%o bereits drei Viertel der Probanden über dem Median der Fehleranzahl, die die Gesamtheit aller Teilnehmer im Nüchternzustand begangen hatten.

Da das Ausgangsniveau, d.h. die Nüchternfahrleistung, innerhalb des Probandenkollektivs stark streute (s. Abb. 21a und b), erschien eine Darstellung der Fahrleistung in relativer Form sinnvoll. Hierzu wurde der im Rahmen der Nüchternfahrt erzielte Punktwert als Bezugswert (100\% entsprechend) festgelegt, um die Veränderung mit steigender BAK ersichtlich zu machen. Eine Verdopplung des im Nüchternzustand erzielten Fehlerpunktwertes entsprach dabei einer relativen Fahrleistung von $50 \%$. In den Abbildungen $22 \mathrm{a}$ und $22 \mathrm{~b}$ ist die Fahrleistung unter Berücksichtigung aller Fahrfehler (Koordination, Konzentration, Reaktion) in relativer Form dargestellt. Die relative Fahrleistung war dabei bereits mit beginnender Alkoholisierung (BAK bis $0,20 \%$ ) signifikant gegenüber der Nüchternleistung reduziert $(p=0,02)$. Bereits bei einer BAK im Korridor zwischen 0,6 und 0,8\%o erreichten mehr als drei Viertel der Probanden nicht mehr ihre persönliche Nüchternleistung. Ab einer BAK von 1,4\% erlangte kein Proband mehr sein individuelles Ausgangsniveau, wie aus Abbildung 22b zu erkennen ist. 


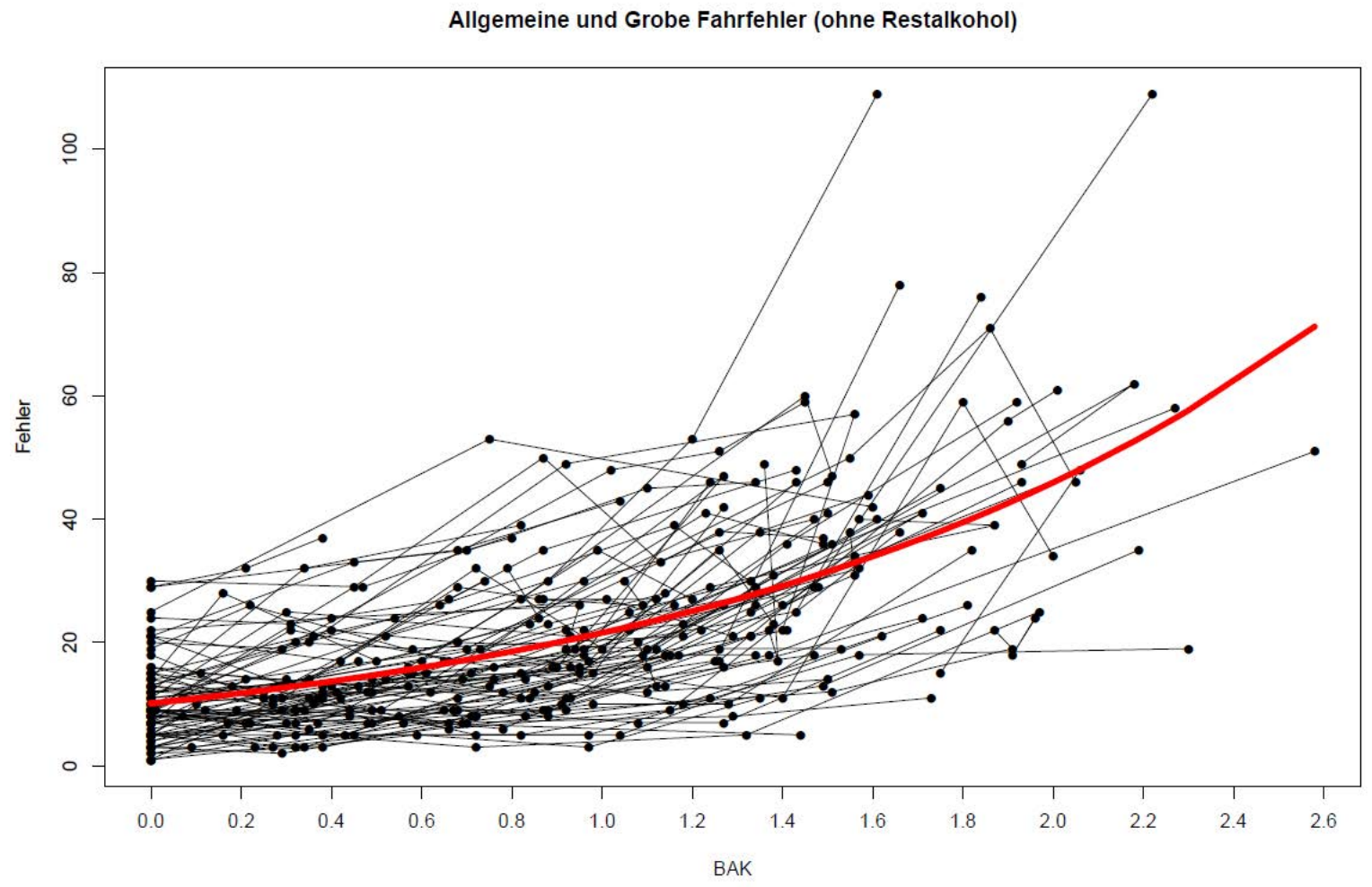

Abbildung 21a:

„Allgemeine und grobe“ Fahrfehler (Koordinationsfehler) in Bezug auf die BAK

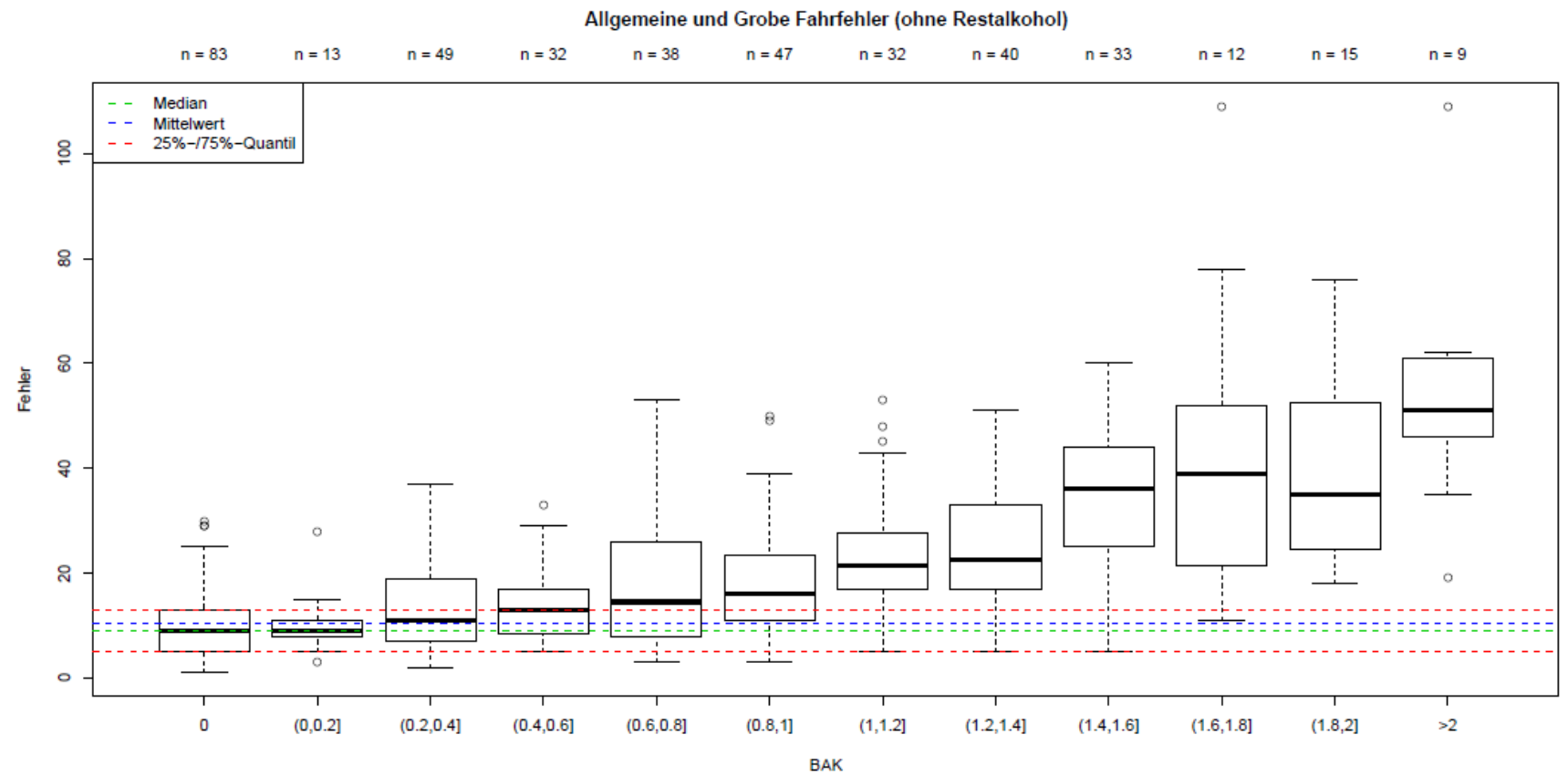

Abbildung 21b:

Darstellung der „allgemeinen und groben“ Fahrfehler (Koordinationsfehler) in Bezug auf die BAK als Box-Plots (runde Klammer: ausschließlich dieses Wertes; eckige Klammer: einschließlich dieses Wertes) 


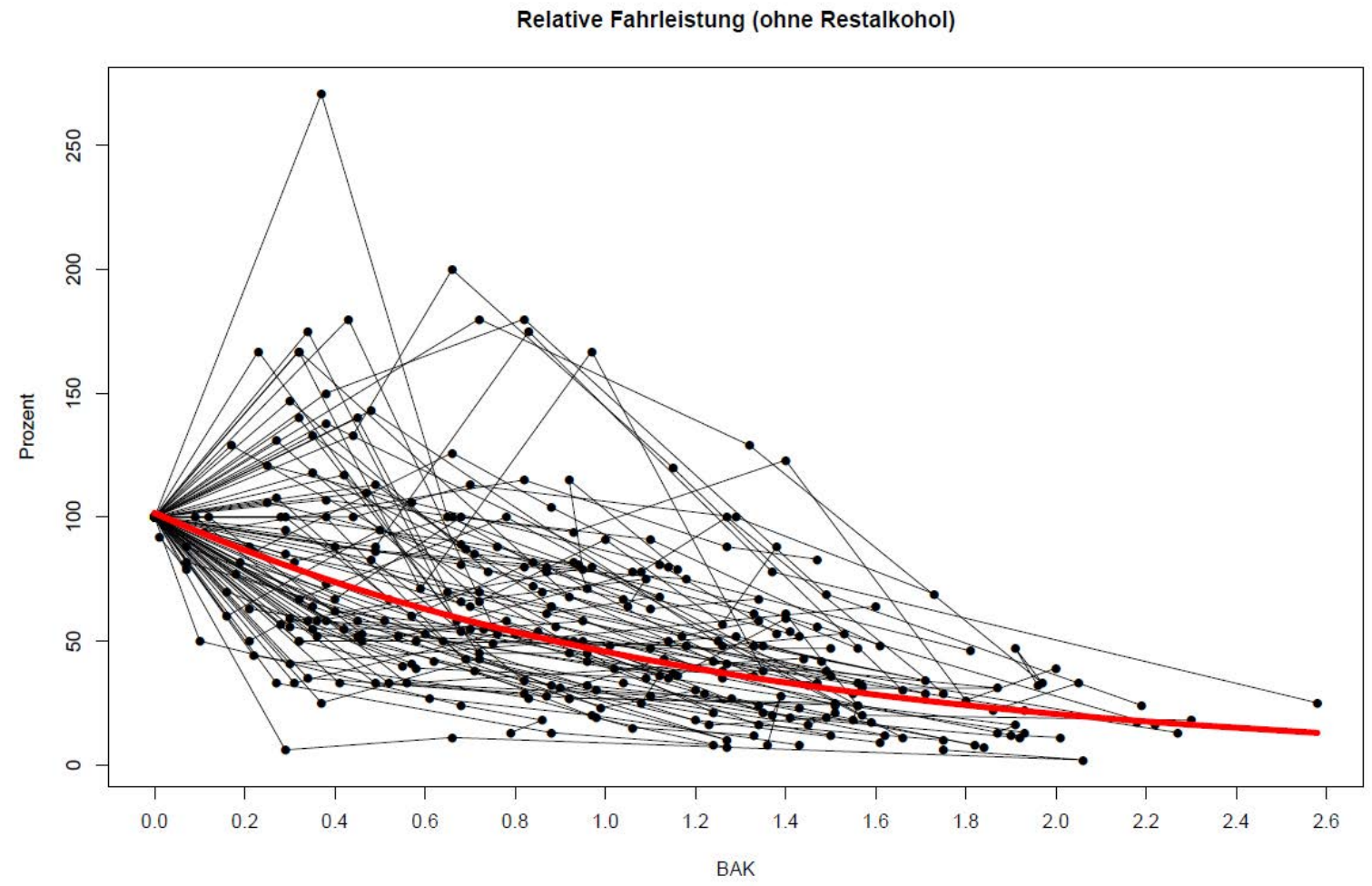

Abbildung 22a:

Relative Fahrleistung unter Berücksichtigung aller Fahrfehler (Koordination, Konzentration, Reaktion) in Bezug auf die BAK

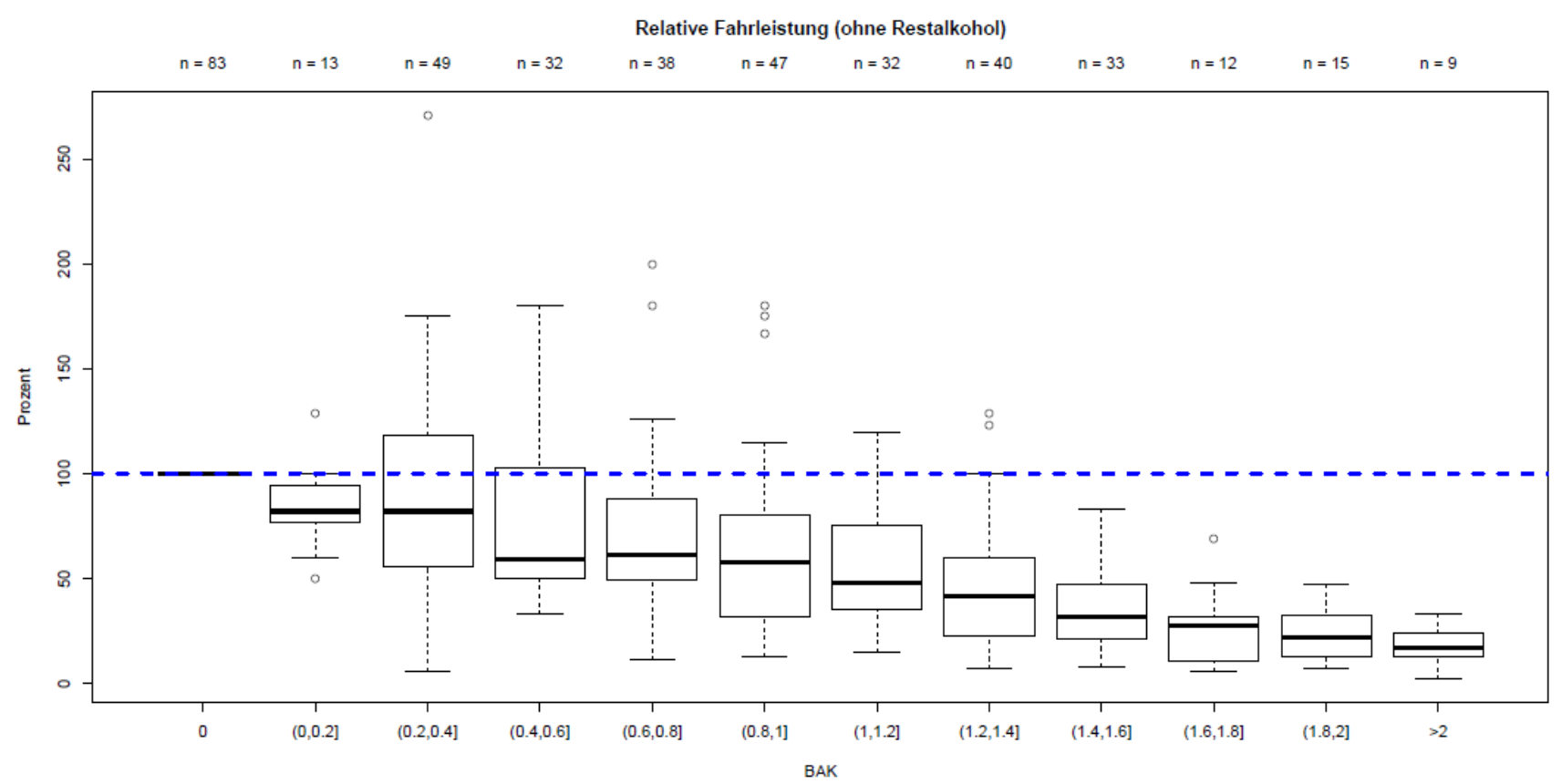

Abbildung 22 b:

Darstellung der relativen Fahrleistung unter Berücksichtigung aller Fahrfehler (Koordination, Konzentration, Reaktion) in Bezug auf die BAK als Box-Plots (runde Klammer: ausschließlich dieses Wertes; eckige Klammer: einschließlich dieses Wertes) 
Beim Vergleich der am Folgemorgen, teils unter Einfluss von Restalkohol begangenen Fahrfehler mit den unter ansteigenden BAK begangenen Fahrfehlern zeigten sich die in Abb. 23 dargestellten Unterschiede. Die BAK-Korridore wurden für diesen Vergleich aufgrund der kleinen Anzahl an Fahrversuchen ( $\mathrm{N}=74)$ relativ breit gefasst. Es liegen im Vergleich zu den entsprechenden Fahrten am Vortag statistisch signifikante Unterschiede für die Korridore 0,00 bis 0,09\% sowie 0,10 bis $0,39 \%$ vor. Konkret bedeutet dies hier, dass auch nach vollständigem bzw. weitestgehendem Alkoholabbau am Folgemorgen signifikant mehr Fehler begangen wurden als bei vergleichbaren BAK am Vortag. Für die Korridore ab 0,40 \%o liegen keine statistisch signifikanten Ergebnisse vor, wobei insgesamt nur sechs Probanden solch hohe Restalkoholkonzentrationen zum Fahrtzeitpunkt aufwiesen.

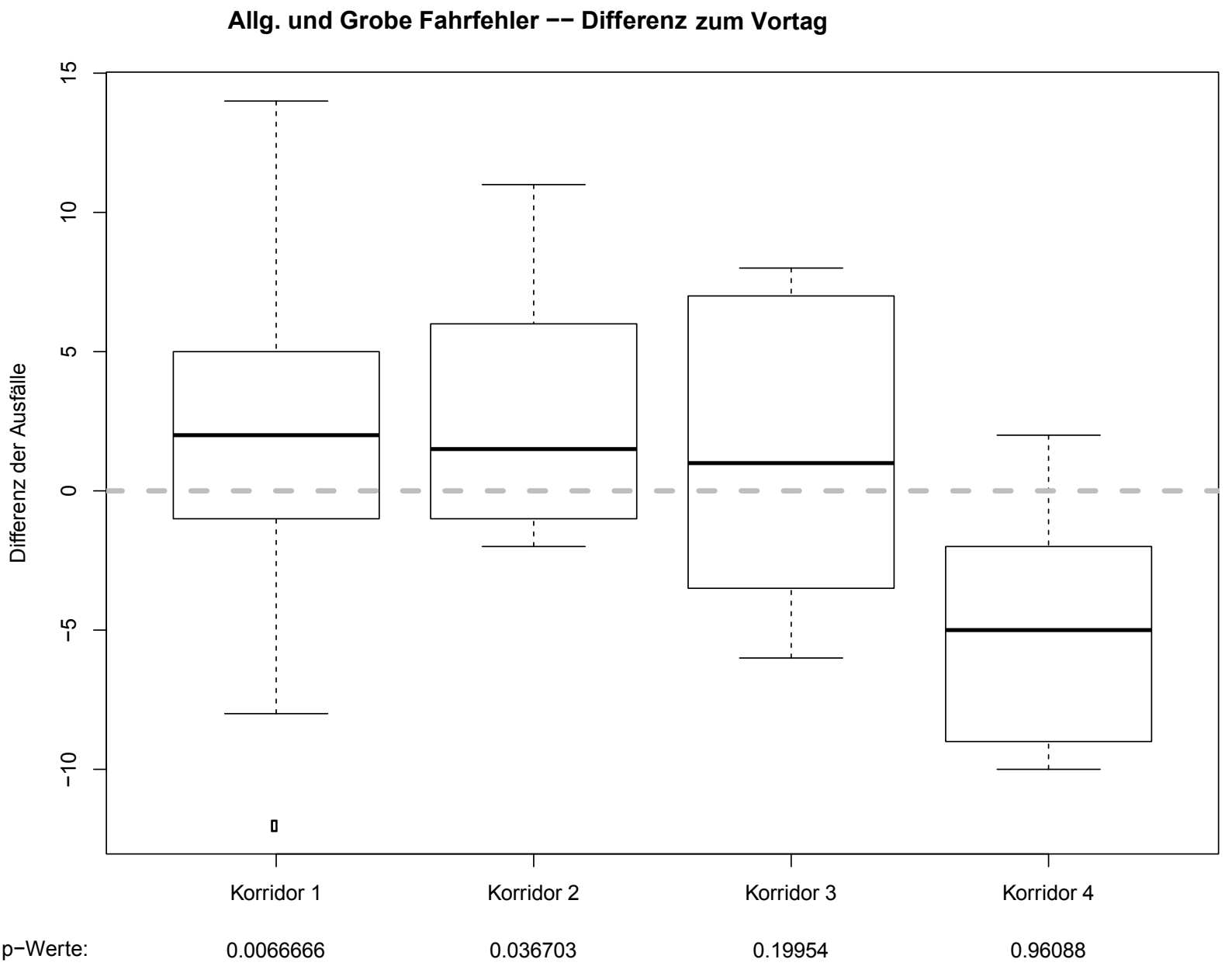

\section{Abbildung 23:}

Allgemeine und grobe Fahrfehler (Koordinationsfehler) am Folgemorgen im Vergleich zum

Vortag (Korridor 1: 0,00 bis $0,09 \%$; $N=38$; Korridor 2: 0,10 bis $0,39 \%$; $N=30$; Korridor 3: 0,40 bis $0,79 \% ; N=5 ;$ Korridor $4:>0,79 \% ; N=1$ ) 


\section{6 Überprüfung der Robustheit}

Die Robustheit der gewählten Methodik zeigt sich, wenn bei abweichender Punktvergabe für Fahrfehler eine gleiche oder ähnliche Abhängigkeit der Leistungseinbuße von der BAK vorliegt. Um dies zu überprüfen, wurden beispielhaft einige der unter 5.5 beschriebenen Fahrfehler mit höheren Punktzahlen belegt.

Folgende Punktwerte wurden alternativ für die jeweiligen Koordinationsfehler vergeben:

1. Linienüberfahrung auf gerader Strecke: 2 Fehlerpunkte.

2. Spur verlassen auf gerader Strecke: 5 Fehlerpunkte.

3. Linienüberfahrung im Kreisverkehr: 2 Fehlerpunkte.

4. Spur verlassen im Kreisverkehr: 5 Fehlerpunkte.

5. Berührung einer Slalomstange/ Pylone nach Stopp an der Ampel: 2 Fehlerpunkte.

6. Umwerfen einer Slalomstange/ Pylone nach Stopp an der Ampel: 5 Fehlerpunkte.

7. Berührung eines Slalomhütchens 2 Fehlerpunkt.

8. Auslassen eines Hindernisses: 5 Fehlerpunkte.

9. Absetzen eines Fußes/ beider Füße: 1 Fehlerpunkt.

10. Anfahrtsschwierigkeiten: 2 Fehlerpunkte.

11. Leichte Schlangenlinien: 3 Fehlerpunkte.

12. Deutliche Schlangenlinien: 5 Fehlerpunkte.

13. Umwerfen bzw. Überfahren der Slalomhütchen 5 Fehlerpunkte.

14. Berührung einer blauen Tonne (Gegenverkehr): 2 Fehlerpunkte.

15. Umwerfen einer blauen Tonne (Gegenverkehr): 5 Fehlerpunkte

Folgende Punktwerte wurden alternativ für die jeweiligen Konzentrationsfehler vergeben:

- Fahren bei gelber Ampel: 2 Fehlerpunkte.

- Fahren bei roter Ampel: 5 Fehlerpunkte.

- Warten bei grüner Ampel ohne ersichtlichen Grund: 1 Fehlerpunkt.

- Überfahrung einer Stopplinie : 5 Fehlerpunkte.

- Für jede von der vorgegebenen Kreisfahrtenanzahl (=3) abweichende Runde: 1 Fehlerpunkt.

- Wort vergessen/ falsch oder nicht abgelesen: 1 Fehlerpunkt.

- Wort teilweise richtig: 0,5 Fehlerpunkte.

Für jeden Reaktionsfehler wurden bei der alternativen Punktwertvergabe 5 Fehlerpunkte vergeben. 
Auch bei alternativer Punktwertvergabe bestätigten sich alle wesentlichen vorherigen Aussagen, wie z.B. die Tendenz zu Leistungsverlust bei zunehmender Alkoholisierung. Wie aus Abbildung 24 ersichtlich wird, sammelten auch hier vereinzelte, stark alkoholisierte Probanden $(>1,6 \%$ ) weniger Koordinationsfehlerpunkte („allgemeine und grobe Fahrfehler“) als andere Probanden im Nüchternversuch. Anhand von Abbildung 25 wird deutlich, dass ab einer BAK von 1,4\%o kein Proband mehr sein persönliches nüchternes Ausgangsniveau erreichen bzw. übertreffen konnte.

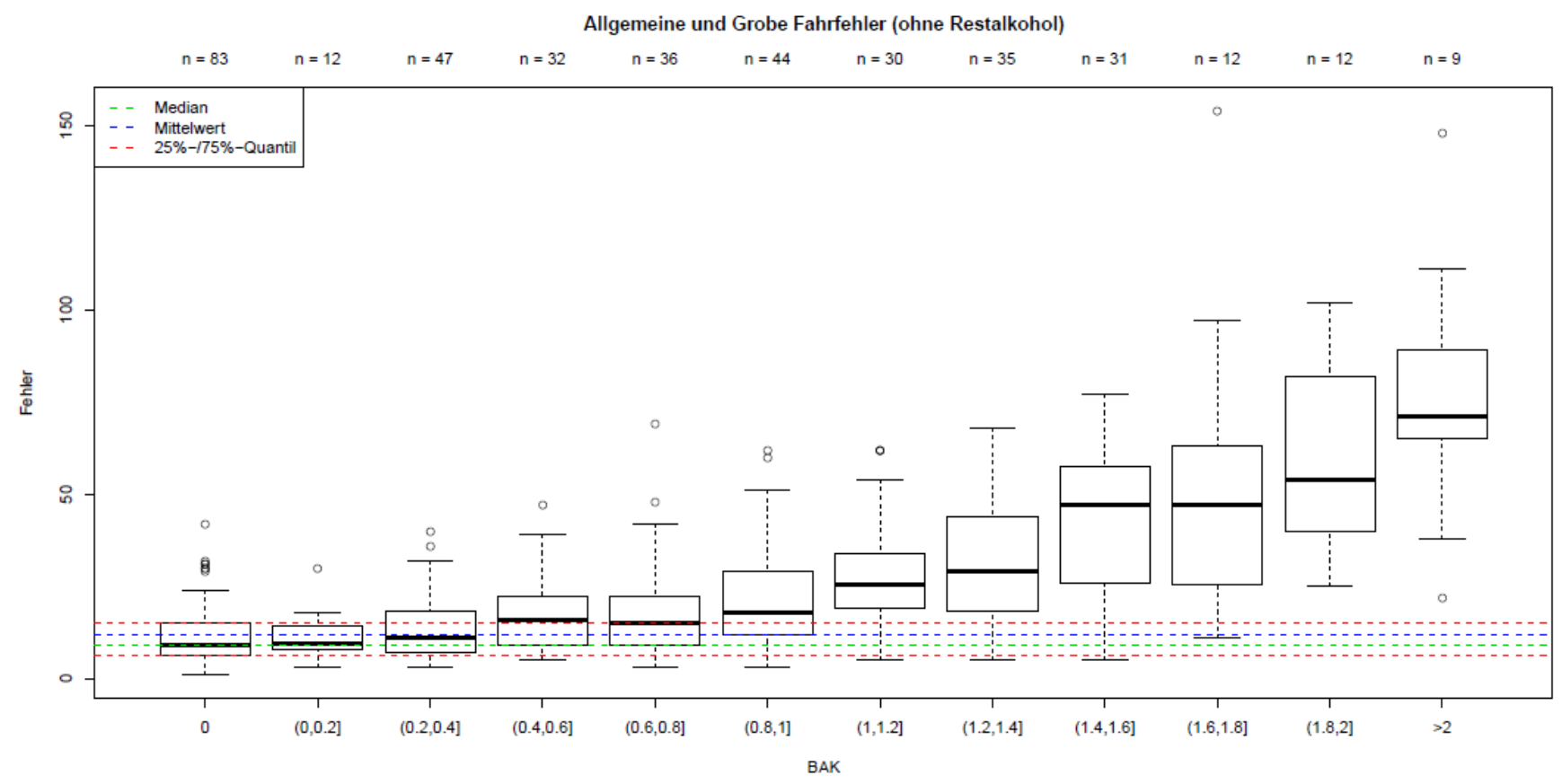

Abbildung 24:

Darstellung der „allgemeinen und groben“ Fahrfehler (Koordinationsfehler) in Bezug auf die BAK als Box-Plots nach alternativer Punktwertvergabe 


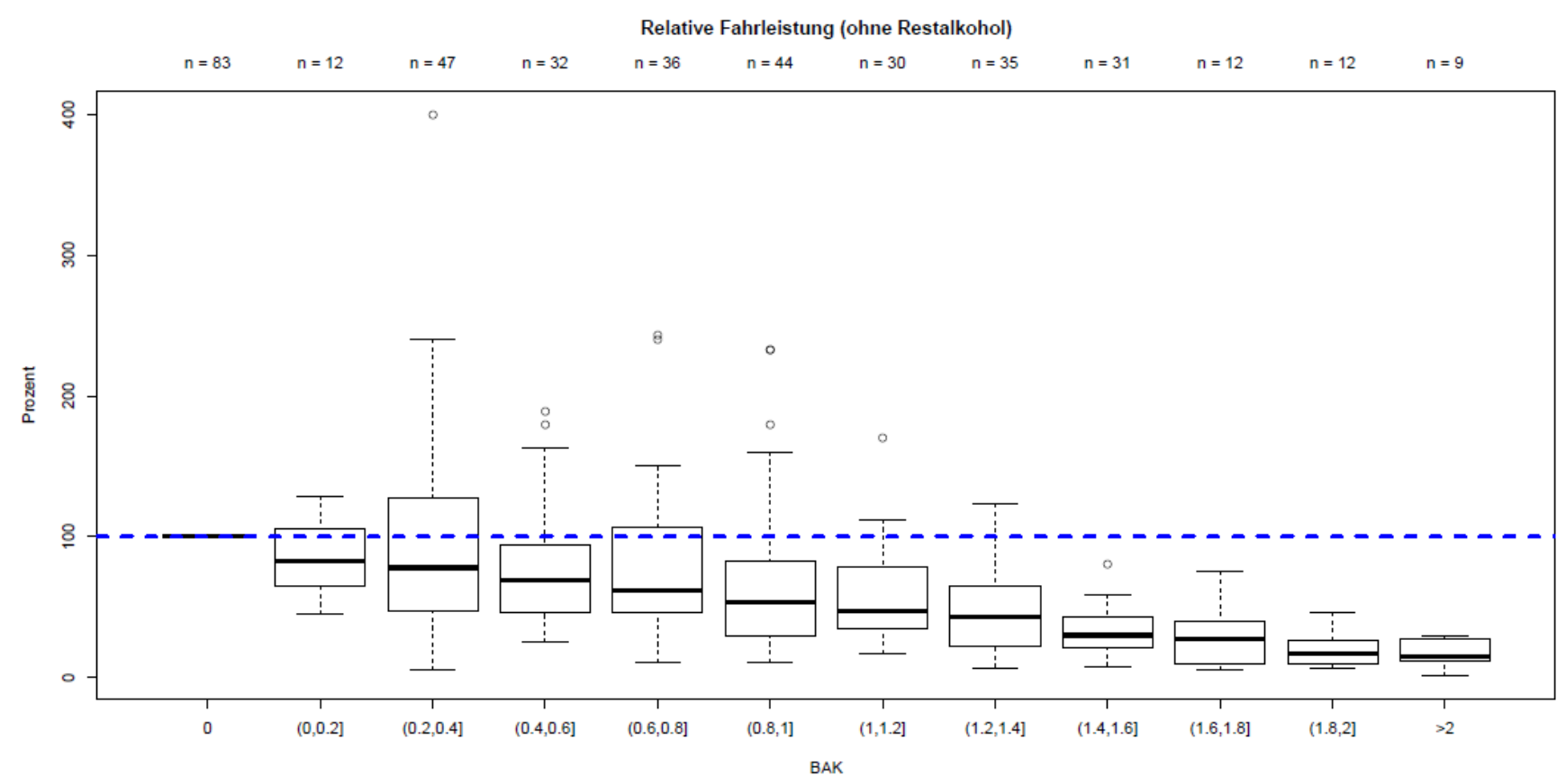

Abbildung 25:

Darstellung der relativen Fahrleistung unter Berücksichtigung aller Fahrfehler (Koordination, Konzentration, Reaktion) in Bezug auf die BAK als Box-Plots nach alternativer Punktwertvergabe

\subsection{Gesamtleistungsvermögen}

Bei der Auswertung des gesamten Leistungsvermögens, das die ärztlich dokumentierten Auffälligkeiten, die Fahrfehler und die Fusionsbreite beinhaltete, zeigte sich, dass der weit überwiegende Teil der Probanden bereits bei einer geringen Alkoholisierung mit BAK bis 0,20\%o leistungsschwächer als im Nüchternzustand war. Spätestens bei einer BAK von 1,4 \%o war ausnahmslos jeder Proband leistungsschwächer als im Nüchternzustand, wobei ab einer BAK von mehr als 1,0\%o kein Proband mehr in der Lage war, das durchschnittliche Ausgangsniveau zu übertreffen (Abb. 26). 


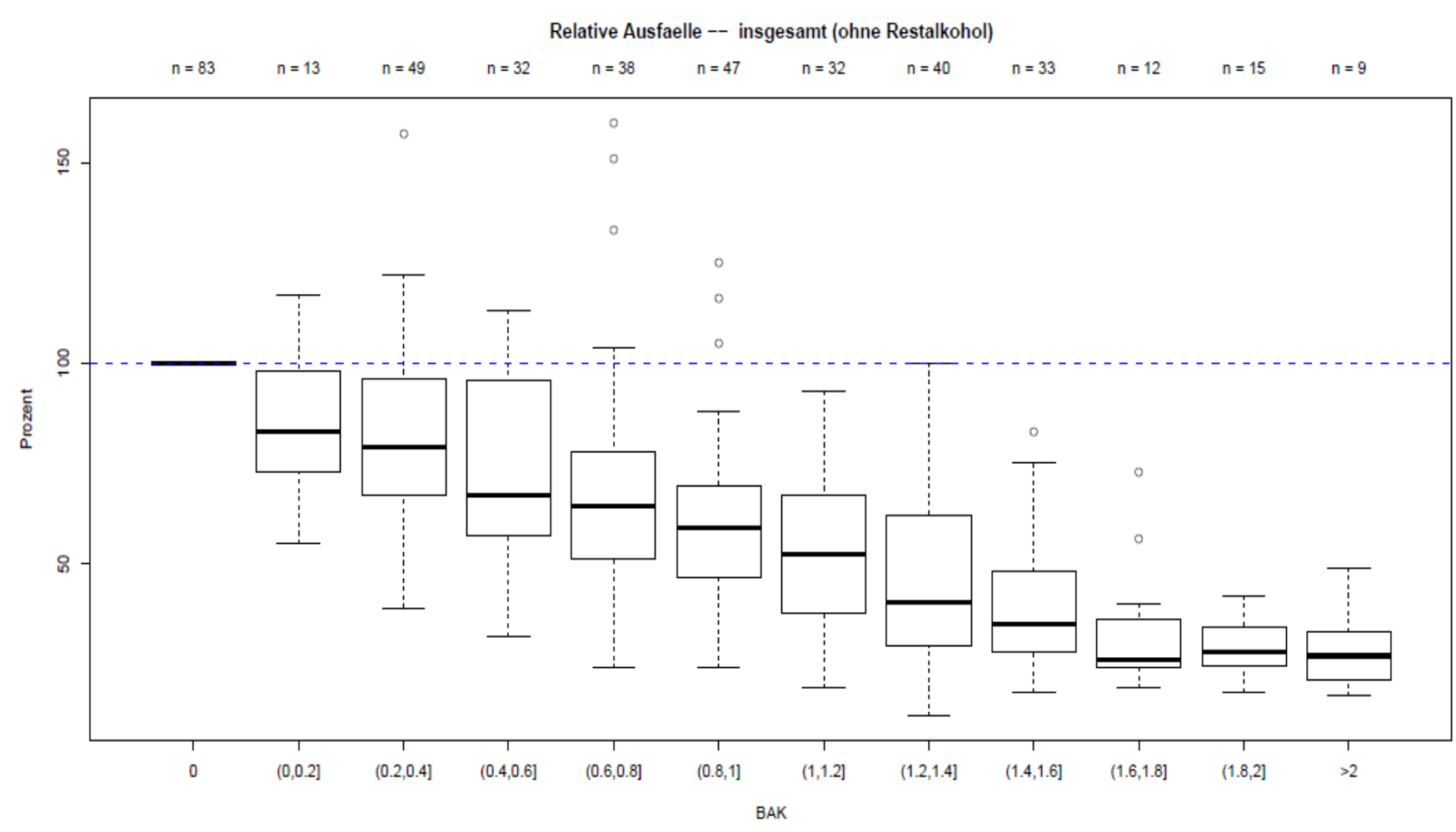

Abbildung 26:

Relatives Gesamtleistungsvermögen in Bezug auf die BAK in Form von Box-Plots

Bei der Beurteilung der oben ausgeführten Ergebnisse der Fahrradversuche in alkoholisiertem Zustand müssen einige grundsätzliche Überlegungen angestellt werden: Der Versuchsaufbau ist sowohl in Bezug auf die Höhe der erreichten Blutalkoholkonzentrationen als auch in Bezug auf den zu bewältigenden Parcours an die Grenzen des ethisch Vertretbaren gegangen. Bei den Probanden handelte es sich - in Analogie zu den von Schewe et al. [1, 2] durchgeführten Fahrversuchen - um ausschließlich gesunde und motivierte Probanden, die sich ganz überwiegend in einem jüngeren Lebensalter befanden. Die Teststrecke und das verwendete Fahrrad durften solange zu Übungszwecken verwendet werden, bis sich der jeweilige Proband auf dem Fahrrad sicher gefühlt und die Fahrstrecke verinnerlicht hatte. Zwar wurde ein zufälliges Ereignis in die Teststrecke eingebaut, das eine komplexe Reaktion des Probanden erforderlich machte, eine echte Gefahr konnte aus Sicherheitsgründen in den Versuchsablauf jedoch selbstverständlich nicht integriert werden. 
Des Weiteren ist bei der Auswertung grundsätzlich die Frage zu stellen, wie man bestimmte Auffälligkeiten bzw. Fahrfehler gewichtet. Bekanntermaßen gefährdet der Radfahrer, zumal wenn er keinen Fahrradhelm trägt, in erster Linie sich selbst und in zweiter Linie fremde Personen. Dennoch ist es selbstverständlich möglich, dass ein Radfahrer einen anderen Verkehrsteilnehmer, z.B. einen anderen Radfahrer oder einen Pkw-Fahrer, in eine Verkehrssituation bringt, in der dieser sich einer riskanten Situation ausgesetzt sieht oder in der es zu einer direkten Kollision kommt. Unter diesem Gesichtspunkt erfolgte von uns die Klassifikation in "grobe“ und „allgemeine“ Fahrfehler, da für die groben Fahrfehler (z.B. vollständiges Verlassen der eigenen, vorgegebenen Fahrspur) die unmittelbare Herbeiführung einer riskanten Verkehrssituation auch für andere Verkehrsteilnehmer angenommen werden kann.

Ein sehr wesentlicher Unterschied zu den von Schewe et al. durchgeführten Versuchen war die Art der Alkoholaufnahme. Während bei Schewe et al. größere Alkoholmengen in standardisierter Form bereits am Vormittag aufgenommen wurden, erfolgte der Alkoholkonsum in hiesigem Versuchsaufbau erst ab dem Nachmittag, wobei sowohl hinsichtlich der Alkoholart und der Alkoholmenge in individueller Geschwindigkeit variiert werden konnte. Ferner war die gleichzeitige Möglichkeit zu einer beliebigen Nahrungsaufnahme gegeben. Bekanntermaßen wird der Alkohol am Vormittag bzw. Morgen schlechter, bei langsamerer Aufnahme hingegen besser vertragen. Die gleichzeitige Nahrungsaufnahme dient dabei zusätzlich einer Verzögerung und Verminderung der Alkoholresorption.

Die Ergebnisse der Radfahrversuche lassen fünf wesentliche Aussagen zu:

- Mit Ausnahme der insgesamt eher selten stattgehabten „groben“ Fahrfehler, bei denen erst ab einer BAK von mehr als $0,8 \%$ eine signifikante Zunahme im Vergleich zum Nüchternzustand vorlag, konnte für die allgemeinen Fahrfehler ebenso wie für die alkoholbedingten Auffälligkeiten, die sich aus dem ärztlichen Bericht anlässlich der Blutentnahmen ergaben, eine signifikante Zunahme spätestens bei BAK im Korridor zwischen 0,21 und 0,40 \%o festgestellt werden. Diese Befunde sind plausibel mit der hierzu vorhandenen verkehrsmedizinischen Literatur in Einklang zu bringen, die besagt, dass bereits bei einer BAK zwischen 0,2 und 0,3\%o relevante Leistungseinbußen vorliegen können [3].

- Wenn man die Fahrleistungen bei steigenden Alkoholkonzentrationen (zu Versuchsbeginn) mit der Fahrleistung am Folgemorgen (Zustand nach erheblicher Alkoholisierung) vergleicht, erkennt man, dass am Folgemorgen statistisch signifikant mehr Fehler begangen wurden. Dies kann einerseits als Beleg für die bekannte Annahme gewertet werden, dass der Schlaf in alkoholisiertem Zustand weniger erholsam ist als im 
Nüchternzustand; andererseits kann der postalkoholische Zustand (,Kater“) auch ohne messbare BAK eben auch dann zu einer relevanten, jedoch nicht mit einer Blutprobe erfassbaren Leistungseinschränkung führen. Interessanterweise fanden sich am Folgemorgen auch im BAK-Bereich bis 0,39\% signifikant mehr Fehler als bei steigenden bzw. zumindest weitgehend stabilen, vergleichbaren BAK am Vortag.

- Mit steigender Blutalkoholkonzentration nahmen die alkoholbedingten Auffälligkeiten in Bezug auf Fahrfehler, die im ärztlichen Bericht dokumentierten Ausfälle und hinsichtlich der nachlassenden Fusionsbreite zu, wobei mit Ausnahme der (nachlassenden) Fusionsbreite ab einer Blutalkoholkonzentration von etwa 1,0\% eine exponentielle Fehlerzunahme zu erkennen war. Auch dies deckt sich mit den Angaben in der Literatur, dass ab einem Promille-Wert von 1,0 bis $1,1 \%$ kein Proband ohne relevante Leistungsbeeinträchtigung ist [3]. Offensichtlich können alkoholbedingte Fahrfehler bei niedrigeren BAK-Werten in der Regel kompensiert werden.

- Die Frage, ob oberhalb einer Blutalkoholkonzentration von 1,6\%o ausnahmslos jeder Proband als fahrunsicher zu bewerten ist, muss auf Grundlage der vorliegenden Ergebnisse - wenn man ausschließlich die Fahrfehler bzw. die relative Fahrleistung unter oben aufgeführter Punktvergabe betrachtet - verneint werden. Hierzu sei auf die Abbildungen 20a und $b$ (Grobe Fahrfehler) und 21a und $b$ (Allgemeine und grobe Fahrfehler) verwiesen, welche besagen, dass vereinzelte Probanden auch bei sehr hohen BAK jenseits der 1,6\% noch unterhalb des durchschnittlichen Fehlerniveaus aller Probanden im nüchternen Zustand lagen. Gleichzeitig ist jedoch festzuhalten, dass ab einer BAK von 1,4 \%o kein Proband mehr in der Lage war, sein individuelles, nüchternes Ausgangsniveau bezüglich der gesamten Fahrfehler zu erreichen oder gar zu übertreffen. 


\section{$7 \quad$ ZUSAMMENFASSUNG}

Die durchgeführten Untersuchungen sollten die Frage beantworten, ab welchem Alkoholisierungsgrad von absoluter Fahruntüchtigkeit auf dem Fahrrad auszugehen ist. Insbesondere war zu klären, ob der derzeit gemäß aktueller Rechtsprechung festgelegte Grenzwert der Blutalkoholkonzentration (BAK) von 1,6\%o zur Annahme einer absoluten Fahruntüchtigkeit geeignet ist. Hierzu wurden praktische Probandenversuche unter möglichst realistischen Bedingungen (z.B. Asphaltboden, verschiedene Witterungsverhältnisse, Alkoholkonsum in geselligem Rahmen) durchgeführt, wobei die von Schewe et al. durchgeführten Fahrversuche erweitert und durch medizinische Untersuchungen ergänzt wurden.

Insgesamt 74 Probanden beiderlei Geschlechts im Alter zwischen 18 und 53 Jahren nahmen an den Versuchen teil, wobei 83 auswertbare Versuchsreihen vorlagen. Für die Versuche lag ein positives Votum der Ethikkommission der Heinrich-Heine-Universität, Düsseldorf vor. Die Fahrten erfolgten unter speziellen Sicherheitsvorkehrungen, wie z.B. Tragen von Fahrradhelm und Motorradschutzkleidung.

Die Versuche wurden mittels Videokameras aufgezeichnet, so dass eine Auswertung mittels der Vergabe von Fehlerpunkten möglich war.

Zusammenfassend ist festzuhalten, dass sich relevante Ausfallserscheinungen bereits bei niedrigen BAK-Werten finden ließen. Die meisten Probanden waren in der Lage, die gröbsten Ausfallerscheinungen während der Fahrversuche bis zu einer BAK von etwa 0,8\% zu kompensieren und grobe Fahrfehler zu vermeiden. Eine solche Kompensation gelang bei der ebenfalls im Rahmen der augenärztlichen Untersuchungen ermittelten Fusionsbreite jedoch nicht; diese nahm mit steigender BAK ab.

Spätestens ab einer BAK von 1,1 \%o nahmen die alkoholbedingten Ausfallerscheinungen und die daraus resultierenden Fahrfehler exponentiell zu. Nur vereinzelte Probanden waren in der Lage, die alkoholbedingten Ausfallerscheinungen während der Fahrversuche auch in sehr hohen BAKBereichen zu kompensieren. Bis zu einem BAK-Wert von 1,6 \% gab es einige wenige Probanden, deren Anzahl Fahrfehler nicht höher war als der Medianwert für alle Probanden im nüchternen Zustand. Spätestens ab 1,4\% konnte allerdings kein Proband mehr seine persönliche Fahrleistung erreichen, die er im nüchternen Zustand gezeigt hatte. 


\section{SUMMARY}

The performed trials were supposed to answer the question, which level of alcoholisation causes „absolute impairment" of riding a bicycle. In particular, we wanted to find out if the valid threshold value of $1.6 \mathrm{~g} / \mathrm{L}$ blood alcohol level (BAL) needs adaptation. Therefore we carried out drinkingcycling trials under conditions as realistic as possible (e.g. asphalt ground, different weather conditions, alcohol consumption in convivial gatherings). The trials performed by Schewe et al. [1, 2] served as a basis and were additionally expanded by new challenges and medical examinations.

A total of 74 test persons of both sexes, aged between 18 and 53 years, participated in the trials. 83 evaluable test series were obtained. The trials were pre-approved by the ethics commitee of the Heinrich-Heine-University, Duesseldorf. Practical trials were conducted under special safety conditions, such as wearing a bicycle helmet and motorcycle protection clothing.

The practical trials were videotaped. The inspection of the videotapes allowed an evaluation by allocation of failure points for certain distinctive features.

From a medicolegal point of view it has to be underlined that relevant alcohol-related deficits can already be found at rather low blood alcohol levels (BAL). Most test persons were able to compensate the most severe alcohol-related deficits up to BAL of around $0.8 \mathrm{~g} / \mathrm{L}$ and avoid severe mistakes during cycling. However the amplitude of fusion as determined during ophthalmologic examination was resistent to compensation mechanisms and decreased with increasing BAL.

At BAL of $1.1 \mathrm{~g} / \mathrm{L}$ alcohol-related deficits and resultant signs of an impairment to drive increased exponentially. Only isolated test persons were able to compensate alcohol-related deficits during the parcours at very high BAL. Up to $1.6 \mathrm{~g} / \mathrm{L}$ BAL, some individuals made not more severe cycling mistakes than the median value for all participants under sober conditions. However, at $1.4 \mathrm{~g} / \mathrm{L}$ BAL, at latest, no one was able to reach his personal cycling performance that he had shown when sober. 
Von Richter BGH i.R. Kurt Rüdiger Maatz

Wie dargelegt, hat sich der Versuchsablauf der vorliegenden wissenschaftlichen Untersuchung eng an die entsprechende Versuchsanordnung der Gutachten von Schewe et al. aus den Jahren 1980 und 1984 [1, 2] angelehnt. Dies erschien wichtig, um einen Vergleich der jetzigen Ergebnisse mit denen jener Untersuchungen zu ermöglichen. Zugleich wurden die Anforderungen an die von den Versuchsteilnehmern zu erbringenden Fahrleistungen verschärft, um den real von Radfahrern zu bewältigenden Verkehrssituationen näher und damit zu einer aussagekräftigeren Beurteilung zu gelangen. Hiernach genügen die Ergebnisse - was der $\mathrm{BGH}$ schon für die früheren Gutachten angenommen hat - den an eine wissenschaftliche Überzeugungsbildung zu stellenden Anforderungen (BGHSt 34, 133, 135).

Zu allererst bestätigt die vorliegende Untersuchung, dass Alkoholisierung auch beim Radfahren eine Gefahr für die Verkehrssicherheit bildet. Und ebenso erweist sich danach, dass die bisher in der Rechtsprechung angenommene untere Grenze möglicher alkoholbedingt „relativer“ Fahrunsicherheit von $0,3 \%$ auch für Radfahrer nach wie vor plausibel ist.

Das aus rechtlicher Sicht vielleicht bedeutendste Ergebnis der Studie ist, dass sich danach eine Absenkung des richterrechtlich festgelegten strafbegründenden „geltenden“ Grenzwerts alkoholbedingter „absoluter“ Fahrunsicherheit von 1,6\%o für Radfahrer ${ }^{1}$ durch die Rechtsprechung nicht begründen lässt. Denn dieser Wert beruht auf der - unter Beachtung des Zweifelsgrundsatzes gewonnenen - Annahme, dass mit an Sicherheit grenzender Wahrscheinlichkeit jeder entsprechende alkoholisierte (Rad)Fahrer fahrunsicher im Sinne des $\S 316$ StGB ist (vgl. BGHSt 21, 157, 160). Diese Annahme ist durch die jetzige Studie schon für den Grenzwert von 1,6\%o als unterem strafbegründenden Wert eher in Frage gestellt, wenn danach zwar bei einer BAK von $\geq 1,4 \%$ sich ausnahmslos jeder Proband als leistungsschwächer als im Nüchternzustand erwies, aber selbst bei einer Alkoholbelastung von $\geq 1,6 \%$ einige - wenn auch nur vereinzelte - Teilnehmer keine relevante Abweichung vom generellen nüchternen

\footnotetext{
BGHSt 34, 133, der darin bestimmte Wert von 1,7 \% setzt sich zusammen aus dem „Grundwert“ von 1,5\% und einem damals noch für geboten erachteten Sicherheitszuschlag von $0,2 \%$; aus den Gründen der Entscheidung BGHSt 37,89, durch die der BGH den Grenzwert „absoluter“ Fahrunsicherheit für Kraftfahrer auf 1,1\%o gesenkt hat, zieht die h.M. den Schluss, dass der Sicherheitszuschlag auch bei dem Grenzwert für Radfahrer nur noch mit $0,1 \%$ anzusetzen ist.
} 
Ausgangsniveau aller Probanden zeigten. Eher könnte dieses Ergebnis sogar nahelegen, dass die Rechtsprechung den Grenzwert nach oben korrigieren müsste. Denn die Rechtsprechung muss ihre Beweisgrundlagen anlassbezogen stets neuen wissenschaftlichen Erkenntnissen anpassen, sofern diese in den einschlägigen Fachkreisen allgemein und zweifelsfrei anerkannt sind.

Diese zuletzt angedeutete Konsequenz könnte für den Gesetzgeber Anlass sein, den strafbegründenden Gefahrengrenzwert in einer Neufassung von $\S 316$ StGB selbst festzuschreiben und dabei an frühere Überlegungen in dem Verfahren zur Einführung dieser Strafvorschrift durch Gesetz von 1964 anzuknüpfen. Ein solcher abstrakter gesetzlich festgelegter Gefahrengrenzwert könnte nach den Ergebnissen der vorliegenden Untersuchung am ehesten bei zwischen 1,4 und 1,6 \%o liegen. Denn bereits bei BAKen ab 1,4 \%o zeigten sich bei den meisten der Probanden relevante Auffälligkeiten und Fahrfehler in einer Häufung und damit ein solch hohes Maß an abstrakter Gefahr, dass eine dahingehende strafbegründende Festlegung weder das Willkürverbot noch den Verhältnismäßigkeitsgrundsatz tangieren würde. Dies würde zudem dem Umstand Rechnung tragen, dass nach der Unfallstatistik der weit überwiegende Anteil, und zwar im Jahr $201275 \%$, der erfassten, an Verkehrsunfällen mit Personenschaden beteiligten alkoholisierten Radfahrer eine Alkoholbelastung von $\geq 1,4 \%$ aufwiesen [4]. Mit einem (unteren) Grenzwert von 1,4\%o würde auch noch der gebotene Abstand gegenüber dem nach der derzeitigen Rechtslage „geltenden“ Grenzwert sog. „absoluter“ Fahrunsicherheit für Kraftfahrer von 1,1\%o (BGHSt 37, 89) gewahrt, der sich aus der gegenüber Kraftfahrzeugen deutlich geringeren Gefährlichkeit für Dritte beim Radfahren in alkoholisierten Zustand und dem bei alkoholisierten Radfahrern im Vordergrund stehenden Risiko, sich selbst zu schädigen, herleitet.

Sollte neben einer weiter gehenden strafrechtlichen Lösung an der Forderung nach Einführung eines bußgeldbewehrten Ordnungswidrigkeitentatbestandes gegen das Radfahren unter Alkoholeinfluss festgehalten werden, müsste der (gesetzliche) Grenzwert wohl am ehesten mit zwischen ca. 0,8 und $1,1 \%$ festgelegt werden:

Für die Einführung eines abstrakten Gefahrengrenzwerts von 0,5\%o für Radfahrer (wie bislang in § 24a Abs. 1 StVG nur für Kraftfahrer) gibt der Fahrversuch nichts her. Dass eine alkoholtoxische Wirkung auch bei solch niedrigen Alkoholisierungsgraden bei den meisten Probanden feststellbar war (etwa als Enthemmung, verdeutlicht durch risikofreudigeres Fahrverhalten), rechtfertigt eine Gleichbehandlung von Kraft- und Radfahrern nicht. Insoweit haben die Fahrversuche keinerlei Hinweis auf eine statistisch relevante Steigerung des Unfallrisikos gegeben, die in diesem niedrigen Alkoholisierungsbereich auch nur generell-abstrakt eine erhöhte Eigenfährdung oder gar Fremdgefährdung begründen könnte. Davon abgesehen, müsste aus 
Rechtsgründen auch hier der - wie ausgeführt - gebotene Abstand zur dem für Kraftfahrzeuge mit ihrem deutlich höheren Gefährdungspotenzial geltenden Wert von 0,5\%o gewahrt werden.

Im Bereich von $\geq 0,8$ bis $1,1 \%$ zeigte die vorliegende Untersuchung dagegen - insoweit in Übereinstimmung mit den Befunden der früheren Untersuchungen von Schewe et al. - eine bereits signifikante Steigerung der verkehrsrelevanten Leistungseinbußen, die von den allermeisten Teilnehmern bei den Fahrversuchen nicht mehr kompensiert werden konnten. Dies würde aus rechtlicher Sicht die Einführung eines entsprechenden Gefährdungstatbestandes rechtfertigen, um dadurch an die Gefährlichkeit von Alkohol beim Radfahren auch unterhalb der für eine Strafbarkeit maßgeblichen Grenze zu gemahnen (vgl. zur Begründung der Einführung von § 24a Abs. 1 StVG BTDrs. 7/133, S. 4).

\section{$10 \quad$ Literatur}

[1] Schewe, G., Schuster, R., Englert, L., Ludwig, O., Stertmann, W.A.: Experimentelle Untersuchungen zur Frage der alkoholbedingten Fahruntüchtigkeit von Fahrrad- und Mofafahrern. BLUTALKOHOL 17, S. 298-328, Lübeck, 1980

[2] Schewe, G., Knoess, H.P., Schaeufele, A., Schuster, R.: Experimentelle Untersuchungen zur Frage der alkoholbedingten Fahruntüchtigkeit bei Fahrradfahrern. BLUTALKOHOL 21, S. 97-109, Lübeck, 1984

[3] Gerchow J: Alkohol im Straßenverkehr. In: Singer MV, Teyssen S, Alkohol und Alkoholfolgekrankheiten. S. 532-540, Springer-Verlag Berlin Heidelberg, 2005

[4] Statistisches Bundesamt: Verkehrsunfälle, Unfälle unter dem Einfluss von Alkohol oder anderen berauschenden Mitteln im Straßenverkehr 2012. S. 23, Wiesbaden, 2013 
Die Realisierung eines solchen Projektes wäre nicht möglich ohne eine sehr große Anzahl von tatkräftigen Helfern, denen an dieser Stelle in alphabetischer Reihenfolge nochmals ausdrücklicher Dank ausgesprochen werden soll:

- Dr. Gernot Brauers: u.a. LC/MS-Untersuchungen.

- Matthias Breithaupt: Kameramann.

- Dr. Torge Brosig: Ärztliche Untersuchungen.

- Dr. Florence Hellen: Ärztliche Untersuchungen.

- Karl-Heinz Jansen und seine Mitarbeiter: u.a. technischer Aufbau .

- Jeannie Jungnitsch: Ärztliche Untersuchungen.

- Holger Klement: Verpflegung, Getränkeausschank.

- Roland Krichel: u.a. Patiententransport.

- Dorothea Krings: Finanzbuchhaltung.

- Claudia Liebelt: u.a. Datensammlung vor Ort.

- Dr. Philipp Messinger: Ärztliche Untersuchungen.

- Karin Monser: Videoanalysen.

- Justin Moody: u.a. Aufbau des Parcours, AAK-Messungen.

- Annette Rickert: u.a. Überwachung der Probanden, Alco-Tests.

- Dr. Wolfgang Settertobulte: Statistische Auswertung.

- Markus Sichau: u.a. praktische Hilfe vor Ort.

- Verena Sichau: u.a. praktische Hilfe vor Ort.

- Irina Steiner: u.a. LC/MS-Untersuchungen, AAK-Messungen.

- Hannes Theen: Kameramann.

- Ethikkommission der HHU.

- Malteser Hilfsdienst, Neuss.

- Multimediazentrum der Heinrich-Heine-Universität.

- Polizei Neuss.

- Straßenmeisterei Meerbusch.

- Technisches Hilfswerk Neuss.

Besonderer Dank gebührt ferner den Probanden, die sich für die Wissenschaft zur Verfügung gestellt haben. 


\section{GDV}

DIE DEUTSCHEN VERSICHERER

\section{Gesamtverband der Deutschen Versicherungswirtschaft e.V.}

Wilhelmstraße 43/43G, 10117 Berlin

Postfach 0802 64, 10002 Berlin

Tel.: 030/20 20 - 50 00, Fax: 030/20 20 - 6000

www.gdv.de, www.udv.de 